\title{
Mast Cells Are Mediators of Fibrosis and Effector Cell Recruitment in Dermal Chronic Graft-vs.-Host Disease
}

\author{
Ethan Strattan ${ }^{1,2}$, Senthilnathan Palaniyandi ${ }^{1}$, Reena Kumari ${ }^{1}$, Jing Du ${ }^{3}$, Natalya Hakim ${ }^{3}$, \\ Timothy Huang ${ }^{1}$, Melissa V. Kesler ${ }^{3}$, C. Darrell Jennings ${ }^{3}$, Jamie L. Sturgill ${ }^{2,4}$ and \\ Gerhard C. Hildebrandt ${ }^{1,2 *}$
}

'Division of Hematology and Blood \& Marrow Transplant, Markey Cancer Center, University of Kentucky, Lexington, KY, United States, ${ }^{2}$ Department of Microbiology, Immunology, and Molecular Genetics, University of Kentucky, Lexington, $K Y$, United States, ${ }^{3}$ Department of Pathology, University of Kentucky, Lexington, $K Y$, United States, ${ }^{4}$ Division of Pulmonary, Critical Care, and Sleep Medicine, University of Kentucky, Lexington, KY, United States

OPEN ACCESS

Edited by:

Anne Mary Dickinson, Newcastle University, United Kingdom

Reviewed by:

Philippe Saas,

INSERM U1098 Interactions Hôte-Greffon-Tumeur \& Ingénierie

Cellulaire et Génique, France Sandeep Kumar Dhanda, La Jolla Institute for Immunology, United States

${ }^{*}$ Correspondence:

Gerhard C. Hildebrandt gerhard.hildebrandt@uky.edu

Specialty section

This article was submitted to Alloimmunity and Transplantation,

a section of the journal

Frontiers in Immunology

Received: 02 July 2019 Accepted: 03 October 2019 Published: 18 October 2019

Citation:

Strattan E, Palaniyandi S, Kumari R, Du J, Hakim N, Huang T, Kesler MV, Jennings $C D$, Sturgill $J L$ and Hildebrandt GC (2019) Mast Cells Are Mediators of Fibrosis and Effector Cell

Recruitment in Dermal Chronic

Graft-vs.-Host Disease.

Front. Immunol. 10:2470. doi: 10.3389/fimmu.2019.02470
Allogeneic hematopoietic stem cell transplant (allo-HSCT) is often used to treat acute leukemia or defects of hematopoiesis. Its widespread use is hampered by graft-vs.-host disease (GVHD), which has high morbidity and mortality in both acute and chronic subtypes. Chronic GVHD (cGVHD) occurs most frequently in skin and often is characterized by pathogenic fibrosis. Mast cells (MCs) are known to be involved in the pathogenesis of other fibrotic diseases. In a murine model of cGVHD after allo-HSCT, C57BL/6J recipients of allogeneic LP/J donor cells develop sclerodermatous dermal CGVHD which is significantly decreased in mast cell-deficient B6.Cg-Kit ${ }^{W-s h} /$ HNihrJaeBsmGlliJ recipients. The presence of MCs is associated with fibrosis, chemokine production, and recruitment of GVHD effector cells to the skin. Chemokine production by MCs is blocked by drugs used to treat cGVHD. The importance of MCs in skin CGVHD is mirrored by increased MCs in the skin of patients with dermal cGVHD. We show for the first time a role for MCs in skin cGVHD that may be targetable for preventive and therapeutic intervention in this disease.

Keywords: GVHD, fibrosis, mast cells, skin, transplant, immunity

\section{INTRODUCTION}

Graft-vs.-host disease (GVHD) has remained the major limiting factor for the success of allogeneic hematopoietic stem cell transplant (allo-HSCT) for decades. Chronic GVHD (cGVHD) is a subtype of GVHD which is a major contributor to patient morbidity and mortality, occurring in about 50 percent of patients after allo-HSCT (1). It is a complex and enigmatic disease which can resemble autoimmune, inflammatory, or fibrotic immune responses, symptoms of which can occur in any organ system. Most frequently involved is the skin, with symptoms often presenting as sclerosis alongside increased collagen deposition (2). Scleroderma-like cGVHD observed in transplant survivors can result in skin thickening and stiffness at localized sites or can manifest over extended areas of the body which can lead to full loss of mobility and entire body encasement.

Incomplete knowledge of the pathogenesis of cGVHD has long hampered therapeutic approaches. cGVHD is associated with risk factors related to acute GVHD and alloreactive donor immune cell responses. Perhaps because of this association, this debilitating disease is currently treated similarly to acute GVHD with the use of immunosuppressive 
and anti-inflammatory approaches, yet responses are not satisfactory. Furthermore, long-term treatment in this manner is associated with poor tolerability and risk of severe and potentially lethal infections (3). Therefore, additional research into targetable effectors in cGVHD is a clinically unmet need.

Mast cells are long-lived, tissue-resident myeloid granulocytes, traditionally defined by their role in allergy through release of proinflammatory mediators such as histamine and heparin. This narrow role has been challenged in recent years, with studies pointing to novel roles for mast cells in wound healing, antivenom, protection against bacterial pathogens, and recruitment of other immune cells (4). Importantly, mast cells have been shown to be necessary for induction of pathogenic fibrosis in artificially-induced transgenic or bleomycin-induced models (57), but this has not been studied in the context of cGVHD pathogenesis. Preliminary research on this topic was performed several decades ago although it has not since been thoroughly investigated (8-12), likely because of the lack of effective models of mast cell deficiency and the need for better murine models of cGVHD. The development of mast cell-deficient mice and refinement of the murine models of allo-HSCT now allows for a more thorough investigation into the role of mast cells in cGVHD pathophysiology (13).

In this study, we use a minor histocompatibility-mismatched murine model of allogeneic transplant to induce cGVHD of the skin by injection of LP/J bone marrow and splenocytes into either $\mathrm{C} 57 \mathrm{BL} / 6 \mathrm{~J}$ (allo-WT) recipients or B6.Cg$\mathrm{Kit}^{\mathrm{W}-\text { sh }} / \mathrm{HNihrJaeBsmGlliJ} \mathrm{MC}$-deficient (allo-MCd) recipient animals. We find that WT mice develop symptoms of dermal cGVHD, while mast cell-deficiency results in markedly reduced symptomology. Further, we show that mast cells are radioresistant and that mast cells are present in allo-WT but not allo-MCd recipients post-transplant. These mast cells correlate with increased chemokine production and immune effector cell infiltration. Mast cell derived chemokine production was inhibited by ibrutinib or ruxolitinib, both of which are used to treat dermal cGVHD in patients.

This study is the first of its kind to pinpoint mast cells in vivo as effectors of fibrosis and effector cell recruitment in dermal cGVHD. Targeting of these tissue-specific cells could allow for reduction of cGVHD symptoms, and we hypothesize that the efficacy of current treatments for cGVHD could be due in part to blockade of mast cell responses in cGVHD target organs.

\section{RESULTS}

\section{Bone Marrow-Derived Mast Cells Survive After Exposure to Ionizing Radiation or Chemotherapy}

Previous literature by several groups indicated that mast cells might be capable of surviving high doses of ionizing radiation, such as that used in conditioning prior to transplant of donor cells $(8,9,14)$. To determine this, we derived mast cells ex vivo from murine bone marrow precursors from both BALB/cJ and C57BL/6J mice. After 4-6 weeks of culture, these cells displayed morphological similarity to mast cells, and at least $97 \%$ displayed the characteristic markers of mast cells, FCeR1a/cKit ${ }^{+/+}$by flow cytometry (Figure 1A).

We then tested the viability and overall number of these cells after they were treated with either 8.5 or $20 \mathrm{~Gy}$ of ionizing radiation. Mast cells from either $\mathrm{C} 57 \mathrm{BL} / 6 \mathrm{~J}$ or $\mathrm{BALB} / \mathrm{cJ}$ mice showed no change from baseline after $96 \mathrm{~h}$ of culture, implying that mast cells are highly resistant to cell death induced by ionizing radiation (Figure 1B). Rather than total body irradiation (TBI), chemotherapy-based regimens are often used clinically. We tested whether mast cells could survive after $72 \mathrm{~h}$ of exposure to busulfan and cyclophosphamide. We saw that mast cells had no changes in count or viability after dosing with these drugs alone or in combination. Doses were picked based on clinically achievable steady-state serum concentrations $(15,16)$. Since TBI is used in almost all murine models, we then tested whether mast cells would remain functional after irradiation. Degranulation in response to FCeR1a receptor crosslinking was measured by CD107a expression on the cell surface. Degranulation was apparent at all doses and was unaffected by ionizing radiation (Figure 1D).

\section{Mast Cell-Deficient Mice Have Improved Outcome and Decreased Skin Fibrosis After Transplant}

Having seen that mast cells are viable and functional after irradiation, we induced cGVHD in vivo through a minor histocompatibility mismatched murine bone marrow transplant model $(\mathrm{LP} / \mathrm{J} \rightarrow \mathrm{C} 57 \mathrm{BL} / 6 \mathrm{~J}) \quad(17)$, illustrated in Figure 2A. Allogeneic bone marrow transplants were performed into both wild type C57BL/6J (allo-WT) and B6.Cg-Kit ${ }^{\mathrm{W}-\mathrm{sh}} / \mathrm{HNihrJaeBsmGlliJ}$ mast cell-deficient mice (alloMCd). Syngeneic transplant recipients did not develop cGVHD and served as a negative controls. At 7 weeks post-transplant, $18.75 \%$ of the allo-WT group had died from cGVHD, while there were no deaths in either the syngeneic or the allo-MCd mice (Figure 2B). Using a previously-published whole-animal GVHD scoring system (18), we saw significant decreases in GVHD scoring in allo-MCd mice as compared to allo-WT mice by week 5 (Figure 2C).

The mice were sacrificed and analyzed after week 7 posttransplant. Syngeneic mice had almost no skin pathology. However, allogeneic transplantation induced severe skin pathology in WT mice, which was significantly ameliorated in allo-MCd mice, as scored by an independent, blinded, boardcertified pathologist [hematoxylin and eosin $[\mathrm{H}+\mathrm{E}]$ staining, Figure 2D]. In the lung, small intestine, liver, and colon, there were no significant pathologic differences between the allogeneic groups (Supplementary Figure 1). In the allo-WT but not the syngeneic or allo-MCd animals, pathology scores significantly correlated with clinical GVHD scores (Figure 2E). Only mice in the allo-WT group displayed clinical symptoms consistent with sclerotic chronic GVHD, including scaly skin, hair loss, and patchy lesioning (19). When skin from these mice was examined histologically by Masson's trichrome staining, fibrotic cGVHD was evident only in the allo-WT group, including excess collagen deposition, hyperkeratosis, epidermal disruption, and significant 


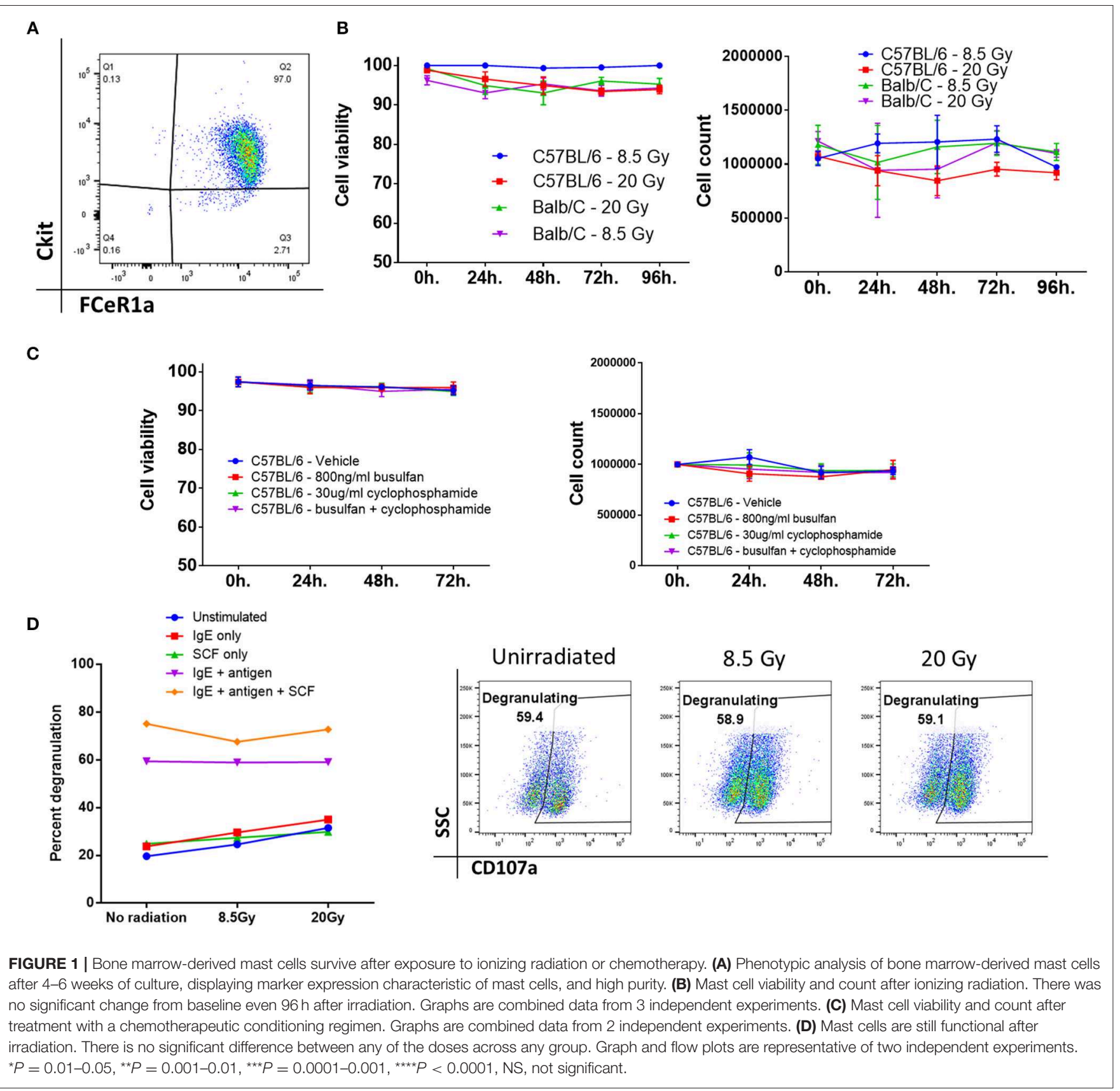

increases in dermal thickness (20), as shown by representative images and the corresponding quantification in Figure 2F. Importantly, no cGVHD was noted in the allo-MCd mice.

We next analyzed RNA extracted from skin of mice from each group by the NanoString Myeloid Innate Immunity panel or by qPCR for IL21 (not included in the NanoString panel). Significant increases in genes associated with skin fibrosis were evident in the allo-WT group, each of which was significantly lowered in the allo-MCd group (Figure 2G) $(21,22)$. Taken alongside the histologic data, this indicates a mast cell-dependent induction of fibrosis through an unknown mechanism.

Since fibroblasts are the main effector cell responsible for collagen deposition and fibrosis, we assessed whether supernatants from mast cells could affect the proliferation of fibroblasts (23). We isolated and cultured fibroblasts derived from the skin of C57BL/6J mice for 2-3 weeks. Following trypsinization, these cells were transferred to a 6-well plate. After $4 \mathrm{~h}$ to allow cells to adhere, there were no differences in count between groups. We then added fresh media or conditioned 

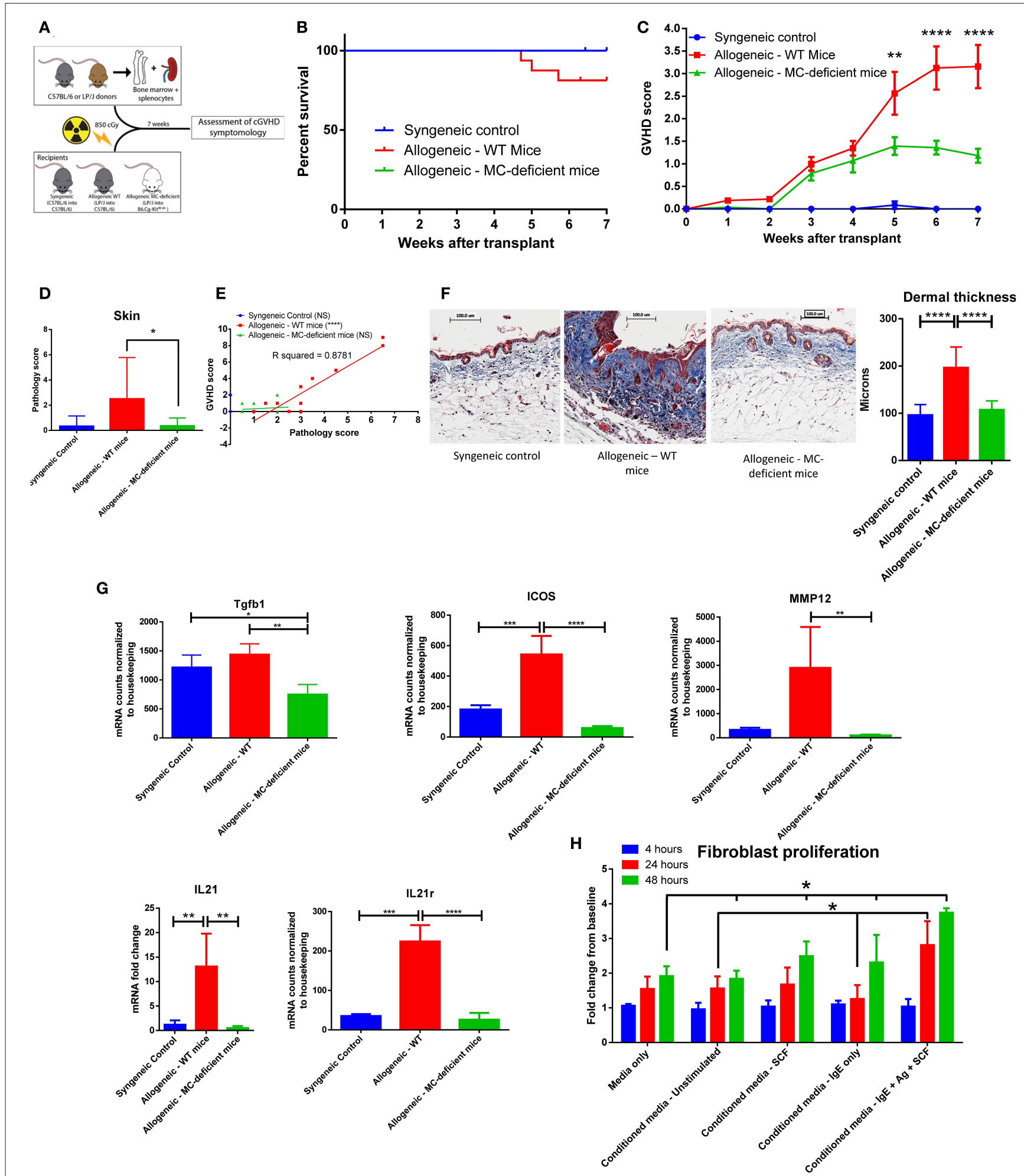

FIGURE 2 | Mast cell-deficient mice have improved outcome and decreased skin fibrosis after transplant. (A) Schematic of transplant to test the effects of mast cell-deficiency in cGVHD. (B) Kaplan-Meier survival curve of syngeneic $(n=6)$, allo-WT $(n=16)$, and allo-MCd $(n=14)$ groups after transplant. The curves are not significantly different, though the syngeneic and allo-MCd groups had no deaths, while the allo-WT group had 3 deaths. Data is combined from two independent transplants. (C) Weekly GVHD scoring of syngeneic $(n=6)$, allo-WT $(n=16)$, and allo-MCd $(n=14)$ groups after transplant. By week 5 , there is a significant reduction in GVHD score in allo-MCd mice as compared to allo-WT mice as measured by a repeated-measures one-way ANOVA. Error bars are the standard error of the mean 
FIGURE 2 | (SEM). (D) Skin pathology score is significantly decreased in the allo-MCd ( $n=16)$ group as compared to the allo-WT group ( $n=14)$. Scoring accounts for damage to the epidermis, dermis, subcutaneous fat, and hair follicles, as well as inflammatory infiltrate, and was performed by a blinded pathologist. Data is combined from two independent transplants. (E) Whole-animal GVHD scoring significantly correlates with skin pathology scoring. R squared and $P$-values were determined by Spearman non-parametric correlation. (F) Representative images and quantification of Masson's trichrome staining of skin from syngeneic $(n=6)$, allo-WT $(n=16)$, and allo-MCd $(n=14)$ groups after transplant. Mice were quantified for dermal thickness according to the height of the blue collagen band across 10-20 different fields per mouse. Images are mice with dermal thickness closest to the mean values for each group. Quantification is combined data from two independent transplants. (G) Genes associated with dermal fibrosis are significantly upregulated in allo-WT animals, as measured by NanoString analysis or qPCR (IL21 only). Samples were skin from syngeneic $(n=3)$, allo-WT $(n=3)$, and allo-MCd $(n=5)$ mice. $(\mathbf{H})$ Supernatants from activated mast cells cause increased proliferation in fibroblasts. $48 \mathrm{~h}$ after plating, supernatants from mast cells stimulated with lgE + antigen $+50 \mathrm{ng} / \mathrm{ml} \mathrm{SCF}$ caused fibroblasts to proliferate significantly more than any other condition. Data is representative of two independent experiments. ${ }^{\star} P=0.01-0.05,{ }^{\star \star} P=0.001-0.01,{ }^{\star \star \star} P=0.0001-0.001,{ }^{\star \star \star \star} P<0.0001$, NS, not significant.

media from mast cells stimulated under various conditions to these cultures and counted them after 24 and $48 \mathrm{~h}$. We saw that after $24 \mathrm{~h}$, fibroblasts cultured in conditioned media from activated mast cells (IgE + antigen + SCF) had grown significantly more than fibroblasts incubated with conditioned media from unstimulated mast cells or from mast cells incubated with $\operatorname{IgE}$ but without antigen. By $48 \mathrm{~h}$ post-plating, fibroblasts cultured in conditioned media from activated mast cells showed a significant increase in cell count compared to all other conditions (Figure 2H). This indicates that products from activated mast cells can induce fibroblast proliferation which could lead to increased fibrosis in vivo.

\section{Mast Cells Are Increased in allo-WT Recipients After Murine Bone Marrow Transplant}

During a bone marrow transplant, the donor stem cells reconstitute and fill the role previously played by the recipient bone marrow. Among these roles are hematopoiesis of lymphoid and myeloid immune subsets, mast cells included (1). Ex vivo, we have shown that mast cells can survive radiation and chemotherapy, but we wanted to determine if mast cells were present post-transplant in vivo; in addition to radiation, recipient-derived mast cells could also be eliminated by alloreactive donor T-cells. Therefore, we used histochemical and fluorescent methods to examine tissue sites where mast cells are tropic, including skin, ear, and trachea, as well as other GVHD target organs (small intestine, lung and liver).

Seven weeks after transplant, organ tissue was stained with toluidine blue and mast cells were counted per high-power field (400x). Skin, ear, and tracheal tissue showed a similar pattern, with low numbers of mast cells in syngeneic animals, higher numbers in allo-WT animals, and low-to-undetectable levels of mast cells in allo-MCd animals (Figures 3A,B, Supplementary Figure 2A). To confirm the identity of the toluidine blue-positive cells, we stained skin slides with avidin conjugated to the Texas Red fluorophore and counted the number of stained cells per high-power field. Avidin has been shown to stain mast cells in a biotin-independent manner $(24,25)$ and skin/ear sections from each animal showed significant correlation between avidin- and toluidine bluepositive cells as well as similar numbers and distribution in the tissue (Figure 3C, Supplementary Figures 2B,C).
Interestingly, mast cells in the skin of the allo-WT group were often seen to be degranulating even at week 7 post-transplant (Figure 3C, Supplementary Figure 2D), indicative of an activated phenotype. In small intestine, lung, and liver, mast cell numbers were low-to-undetectable and there were no differences between groups (data not shown). Findings were verified by a blinded, independent researcher. To confirm our finding in the skin, we returned to our NanoString data set, where we found that mast cell-specific genes including chymase, tryptase, FCeR1a, and Cpa3 were expressed at extremely low levels in the skin of allo-MCd mice (Figure 3D).

\section{The Dermal Environment of allo-WT Mice Is Enriched in Chemokine Signaling Compared to allo-MCd Mice}

Our findings demonstrate a difference in GVHD scoring and skin pathology, yet given the pleotropic effects of mast cells in the immune system we next decided to explore their functional role. We examined immune cells in the spleen and saw no differences in proportion or count in myeloid or lymphoid subsets (Supplementary Figure 3). To examine more local factors, skin from animals from each group was analyzed using the NanoString Myeloid Innate Immunity panel. When we looked at the subset of genes overexpressed in allo-WT vs. allo-MCd by PANTHER pathway analysis (26), we found only one pathway to be statistically significant, an approximately 5 -fold enrichment for the "Inflammation mediated by chemokine and cytokine signaling pathway" (PANTHER pathway number P00031, Supplementary Figure 4A). Cytokine levels were found to be expressed at low levels in both skin and serum and were largely unchanged between groups (Supplementary Figures 4B,D).

Further analysis was performed using the NanoString nSolver software which clusters genes by pre-annotated pathways. Sorting on "Chemokine signaling genes," heatmap analysis demonstrated an overall trend toward increased dermal expression of genes involved in these pathways in allo-WT mice, with lower expression in allo-MCd mice (Figure 4A). We further examined expression of specific chemokine transcripts. CCL2, CCL3/CCL4 (which encode MIP-1a and MIP-1b, respectively), and CCL5 all demonstrated significant upregulation of transcript in the skin in alloWT mice (Figure 4B). When we measured protein levels 


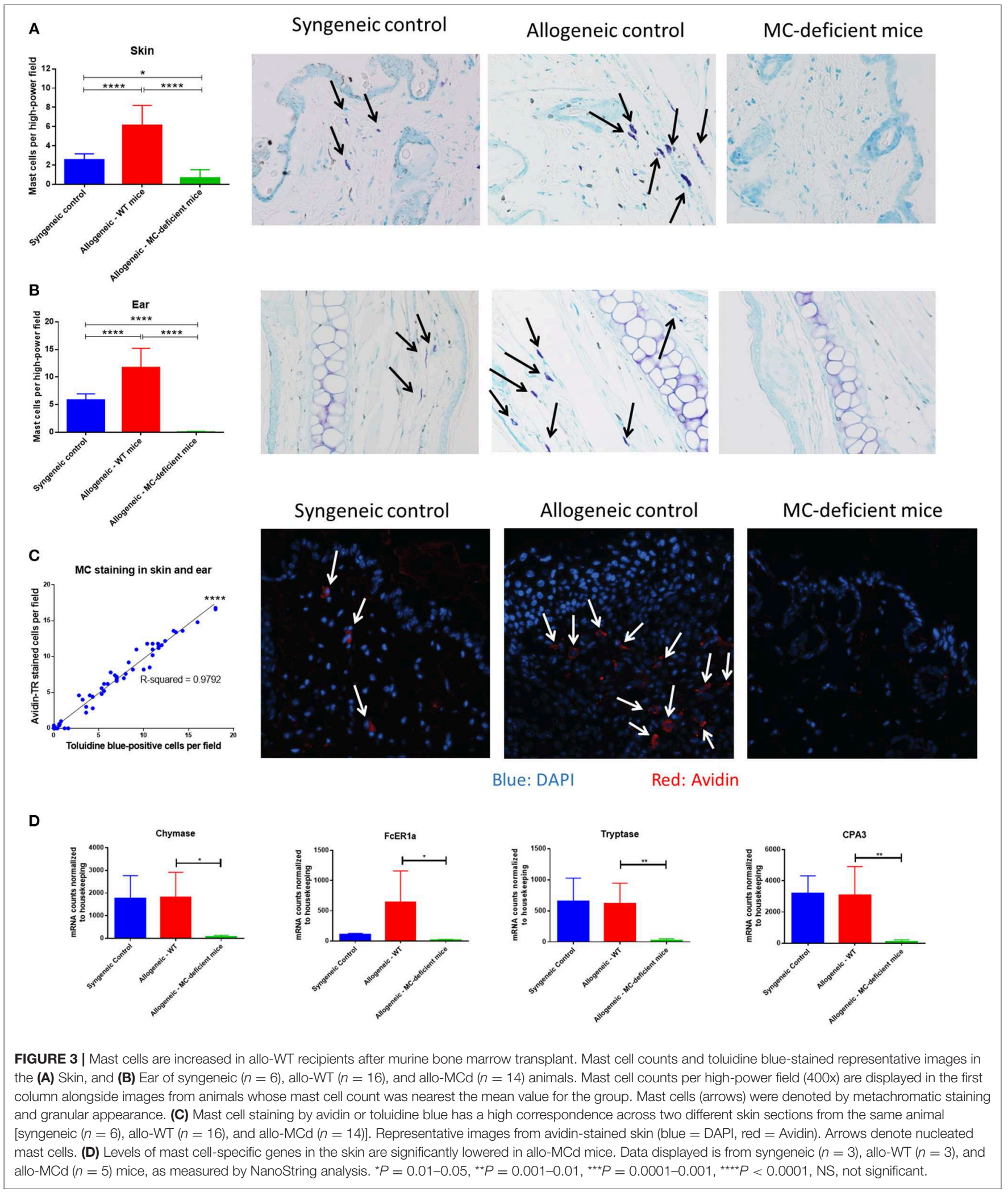

of these chemokines by cytometric bead array, we saw an extremely similar expression pattern, with all 4 chemokines upregulated in allo-WT, while syngeneic and allo-MCd were not significantly different (Figure 4C). Intriguingly, the levels of these chemokines in the allo-WT group were all significantly correlated with their overall GVHD score (Figure 4D). All of 


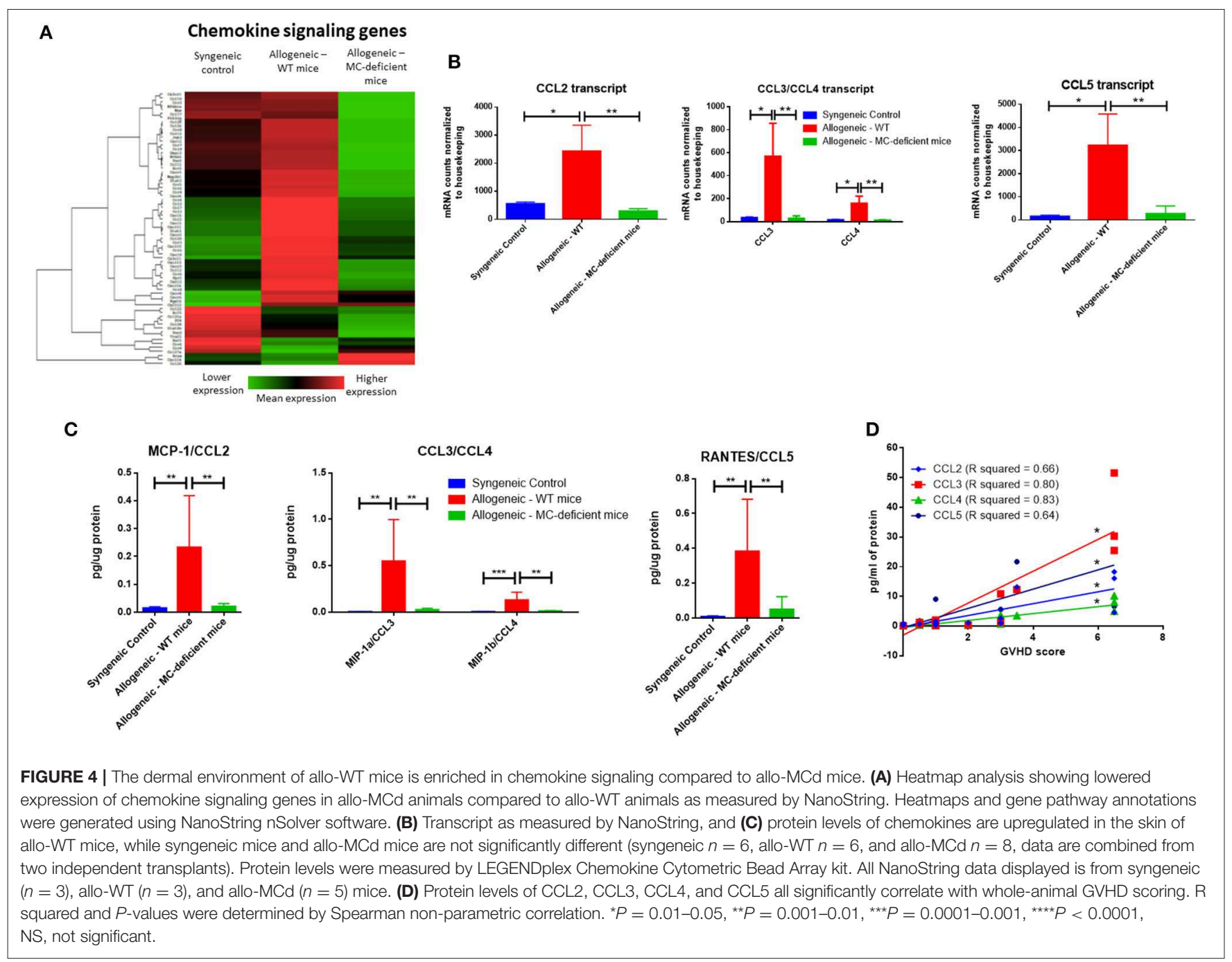

these chemokines can be chemotactic for T-cells, the canonical GVHD effector cell $(27,28)$. Additionally, they are capable of recruiting monocytes, macrophages, or other myeloid cells, many of which are implicated in CGVHD pathogenesis and fibrotic disease.

\section{Immune Infiltration and Activation Are Ameliorated by Mast Cell Deficiency}

Given the inflammation and increased chemokines evident in the skin of our allo-WT mice, we wanted to determine whether this resulted in increased immune infiltration and tissue damage. Indeed, when we examined $\mathrm{H}+\mathrm{E}$-stained skin sections from allo-WT mice, epidermal dysregulation, and an obvious dermal immune infiltrate was visible (Figure 5A, quantified in Figure 5B). Animals from the syngeneic and allo-MCd groups displayed equally low levels of immune infiltrate. The immune infiltrate was characterized as to its hematopoietic lineage and was found to be predominantly lymphocytic in all three groups (Figure 5C). Accordingly, there was no significant difference across all groups in eosinophil or neutrophil count in $\mathrm{H}+\mathrm{E}$-stained skin sections, nor was there a difference in CD19 transcript by qPCR (Supplementary Figure 3C). All characterization and counting on $\mathrm{H}+\mathrm{E}$ slides were performed by an independent, board-certified pathologist.

T-cells in the skin, as measured by immunohistochemistry against $\mathrm{CD} 3$, were upregulated from allo-MCd to allo-WT animals (Figure 5D). This slight but significant increase in cellularity was seen alongside increased gene expression of markers of T-cell activation including CD69 and VAV1, as well as markers of costimulation such as CD80 and CD86 (Figure 5E). It seems that while the level of T-cell infiltration is not hugely different, activation and the costimulatory milieu in the dermal environment may enhance their effects.

\section{Mast Cells Produce Chemokines Upon Stimulation Which Are Blocked by Drugs Used in Treatment of cGVHD}

Mast cells are known to be able to produce a wide variety of mediators, as reviewed excellently by Mukai et al. (29). As shown in Figure 4D, chemokine production seems to be a major factor 
in the pathogenesis of sclerodermatous GVHD in this model. Therefore, we wanted to test whether mast cells were capable of producing these same cytokines, assayed ex vivo using bone marrow-derived mast cells cultured from C57BL/6J mice.

Indeed, we saw that upon stimulation with multiple methods of mast cell activation (IgE/antigen crosslinking or IgE/antigen + IL-33), mast cells produced significant amounts of CCL2, CCL3, and CCL4. Importantly, treatment with ibrutinib, a BTK inhibitor, or ruxolitinib, a Jak1/Jak2 inhibitor, reduced mast cell production of these chemokines in a dose-dependent manner (Figures 6A-C). Treatment with imatinib or fingolimod did not reduce mast cell chemokine production even at the highest doses tested (Supplementary Figure 5A). Drug dosing had no effect on cell viability (Supplementary Figure 5B). While CCL5 was the other chemokine significantly upregulated in the skin of alloWT mice (Figure 4D), we saw no CCL5 production from mast cells in this context, though previous papers from our group have pointed out donor-derived T-cells as the primary producers of CCL5 in the post-transplant setting (30).

\section{Mast Cell Numbers Are Increased in the Skin of Patients With Dermal Manifestations of cGVHD}

To further examine this phenomenon in the context of human patients with cGVHD, we obtained approval from the University of Kentucky Institutional Review Board for studies on banked, deidentified human samples (see methods for details). We used the University of Kentucky Biospecimen Procurement and Translational Pathology Shared Resource Facility (BPTP SRF) to identify skin biopsies from allo-HSCT recipients who were diagnosed with chronic GVHD and where histologic changes were consistent with dermal graft-vs.-host disease. Patient characteristics are included in Table 1. GVHD prophylaxis and antibiotic regimens were unable to be determined from the available anonymized patient data. Samples from 5 cGVHD patients were stained with Masson's trichrome alongside skin samples from patients without cGVHD. Epidermal thickness was measured by Masson's trichrome staining and found to be increased due to collagen deposition, consistent with cGVHD symptomology (Figure 7A). We identified levels of tryptasepositive cells in the skin by immunohistochemistry in order to quantify the number of mast cells present in these samples. Tryptase-positive cells were primarily located near the dermalepidermal junction and were significantly increased in cGVHD patients as compared to normal skin samples as measured by Aperio (Figure 7B). Quantification was verified by a blinded independent researcher. Representative images for the staining are shown in Figure 7C. These data correlate with our findings that mast cells may be important in the pathogenesis of dermal chronic graft-vs.-host disease.

\section{DISCUSSION}

Pathogenic fibrosis is a characteristic of many diseases in the developed world, with up to $45 \%$ of all deaths associated with symptoms of chronic fibrosis (23). Understanding the mechanisms behind fibrosis and developing new treatments is a major public health concern. As in other fibrotic diseases, treatment of cGVHD is hampered by incomplete knowledge of the mechanisms driving disease pathogenesis. Many of the current treatments, such as immunosuppressive steroids, do not bring about the desired patient outcomes and are unsuited for the long-term course of treatment often required in cGVHD. In this study, we demonstrate a novel role for mast cells in the pathogenesis of dermal cGVHD and show the first evidence of mast cell involvement in vivo, which could open new paradigms in the treatment of fibrotic cGVHD.

Our data demonstrating mast cell viability and functionality after radiation and chemotherapy confirm several earlier studies showing similar results (Figures 1B-D) (14, 31). This radioresistance of mast cells is striking in contrast to many other immune cell types, which are considered to be depleted during the conditioning phase of allo-HSCT (19). Additionally, MC resistance to alkylating agents is perhaps not entirely surprising. They express high levels of aldehyde dehydrogenase, which is known to inhibit the activity of alkylating agents, and divide slowly, if at all (32-34). Taken together, this implies that the mast cell is a conditioning-resistant, mature, immune effector cell, which remains functional, and that has tropism toward the cGVHD target organs. It is therefore perhaps unsurprising that there was such a dichotomous difference in cGVHD symptomology in vivo between the allo-WT and allo-MCd groups in our murine model of allogeneic transplant. Using the $\mathrm{LPJ} \rightarrow \mathrm{C} 57 \mathrm{BL} / 6 \mathrm{~J}$ model of murine cGVHD in both WT C57BL/6J

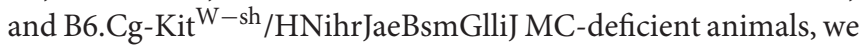
showed evidence for reductions in whole-animal GVHD scoring, skin pathology, and in dermal thickness and fibrosis in the alloMCd mice. This correlated with decreases in transcript level of genes associated with fibrosis in the dermal environment (Figures 2A-G) (21, 22). Additionally, our in vitro evidence demonstrated that mast cells are capable of producing factors that induce proliferation in skin-derived fibroblasts, which are responsible for collagen deposition (Figure 2H). Mast cells are known to be one of the major sources of fibroblast growth factor, which could explain this phenomenon (35).

Previous work by Leveson-Gower et al. has shown that the presence of mast cells is beneficial in the context of acute GVHD through mast cell production of anti-inflammatory factors, such as IL-10 (36). However, that study was performed in a $\mathrm{C} 57 \mathrm{BL} / 6 \rightarrow \mathrm{BALB} / \mathrm{c}$ model of acute GVHD, where defining characteristics of the disease include acute inflammation and early alloreactive damage to the tissues, along with rapid disease onset, progression and mortality from acute GVHD. In contrast, chronic GVHD is often defined by inappropriate and prolonged inflammation alongside anti-inflammatory "wound healing" responses and fibrosis (37). Therefore, we believe that the mast cell, while beneficial in the inflammatory phase of aGVHD, ultimately becomes detrimental in cGVHD through its ongoing anti-inflammatory activities resulting in collagen deposition and pathogenic fibrosis.

These data fit with a study by Levi-Schaffer et al., which demonstrated that mast cells are activated by splenic supernatants from mice with cGVHD, as well as their work 


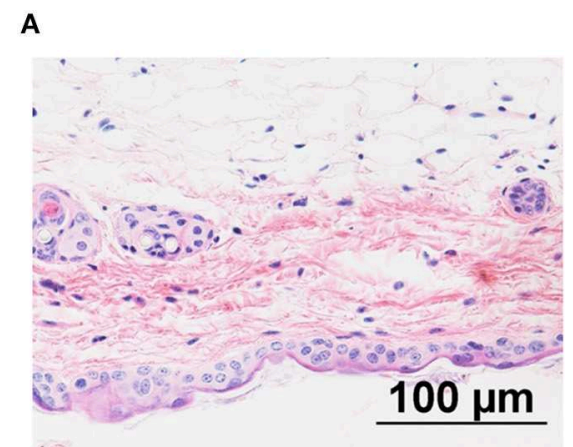

Syngeneic control

B

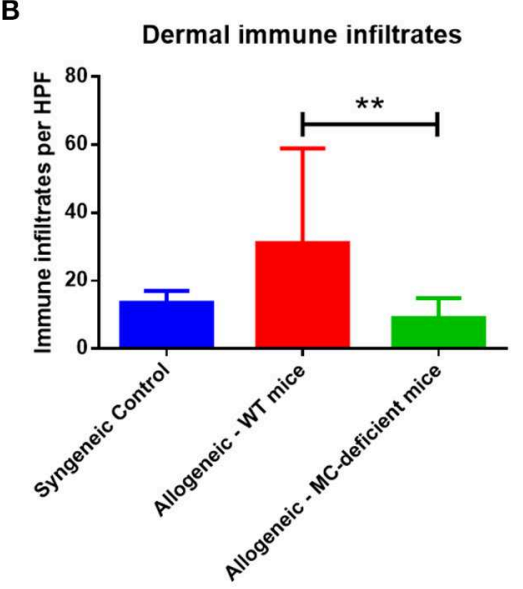

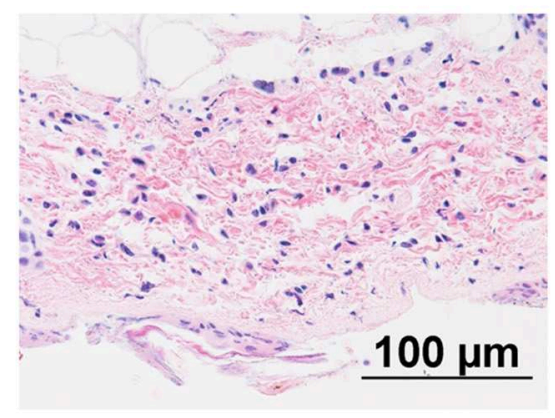

Allogeneic - WT mice

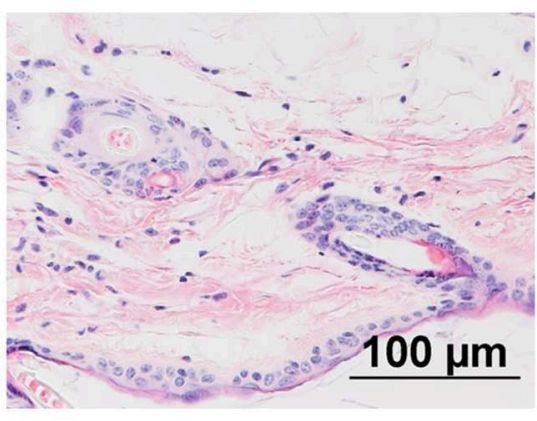

Allogeneic - MC-deficient mice

C
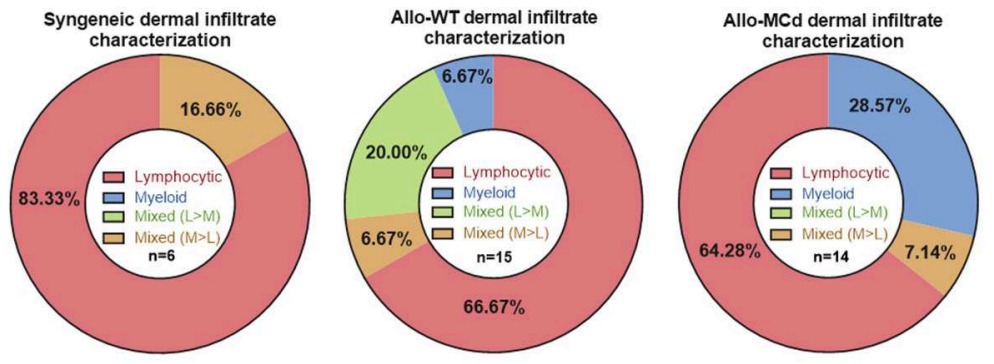

D

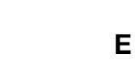

E
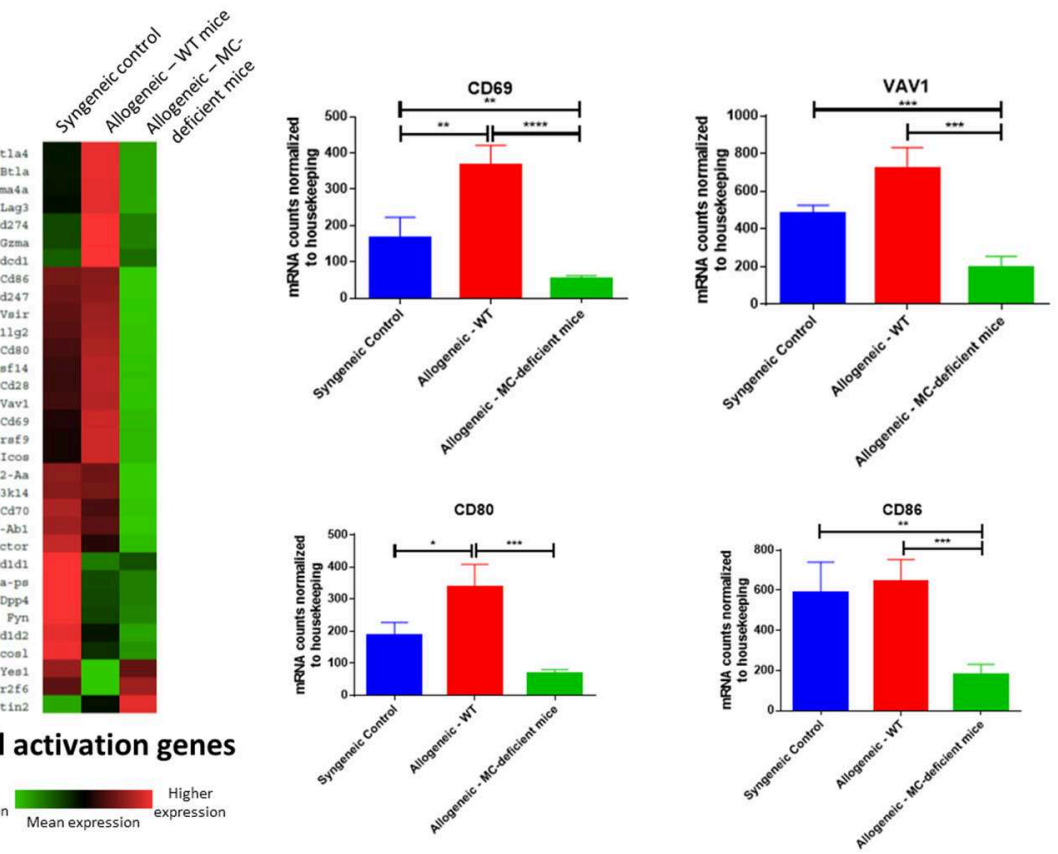

FIGURE 5 | Immune infiltration and activation in the skin are ameliorated by mast cell deficiency. (A) Representative images from $\mathrm{H}+\mathrm{E}$ staining show an increased immune infiltrate and tissue damage in skin from allo-WT animals, while syngeneic and allo-MCd animals have a similar phenotype. In allo-WT animals, epidermal dysregulation and an obvious dermal immune infiltrate is visible. (B) Quantification of (A). (C) The immune infiltrate was characterized as to its hematopoietic lineage by morphology and was found to be predominantly lymphocytic in all three groups. (D) Immunohistochemistry against CD3 demonstrates a significant increase in 
FIGURE 5 | T-cell infiltration in the skin of allo-WT as compared to allo-MCd animals. Slides were digitally scanned using the Aperio ScanScope XT, then quantified for DAB chromogen staining across the whole tissue using the Positive Pixel Count V9 algorithm. (E) Heatmap and gene-level analysis demonstrates significant increases in genes involved in the T-cell activation pathway in allo-WT as compared to allo-MCd animals as measured by NanoString. This indicates that despite a slight but significant increase in T-cells, activation status of those cells widely differs between groups. Heatmaps and gene pathway annotations were generated using NanoString nSolver software. NanoString data displayed is from syngeneic $(n=3)$, allo-WT $(n=3)$, and allo-MCd $(n=5)$ mice. ${ }^{*} P=0.01-0.05$, ${ }^{* *} P=0.001-0.01$, ${ }^{\star \star \star} P=0.0001-0.001,{ }^{\star \star \star \star} P<0.0001, \mathrm{NS}$, not significant.

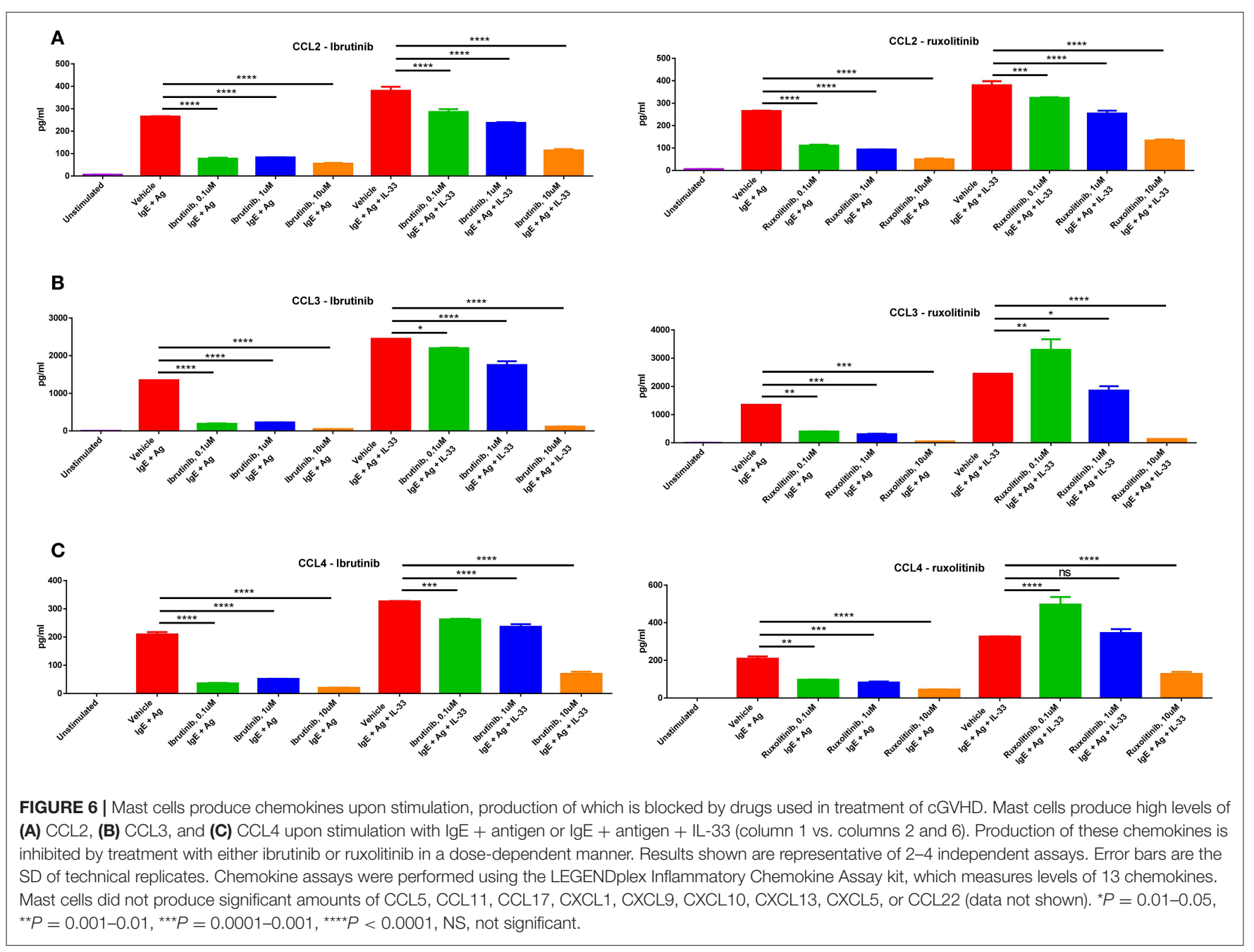

TABLE 1 | Patient characteristics.

\begin{tabular}{|c|c|c|c|c|c|c|}
\hline & $\begin{array}{l}\text { Median age } \\
\text { (range) }\end{array}$ & Gender & $\begin{array}{l}\text { Gender mismatched } \\
\text { donor }\end{array}$ & \multicolumn{3}{|c|}{ Conditioning regimen } \\
\hline & & $F(1 / 5)$ & & & & \\
\hline \multirow[t]{2}{*}{ Non-GVHD patients } & $50(36-60)$ & $M(2 / 4)$ & NIA & & NIA & \\
\hline & & $F(2 / 4)$ & & & & \\
\hline
\end{tabular}

Characteristics of patients used in the study were provided by the UK Biospecimen Procurement and Translational Pathology Shared Resource Facility.

showing that fibroblasts from mice with cGVHD enhance proliferation of connective tissue-derived mast cells $(10,12)$. Taken together with our data showing that mast cell products can stimulate proliferation of fibroblasts (Figure $\mathbf{2 H}$ ) this could indicate a positive feedback loop in cGVHD wherein mast cells and fibroblasts are able to induce proliferation in the other cell 

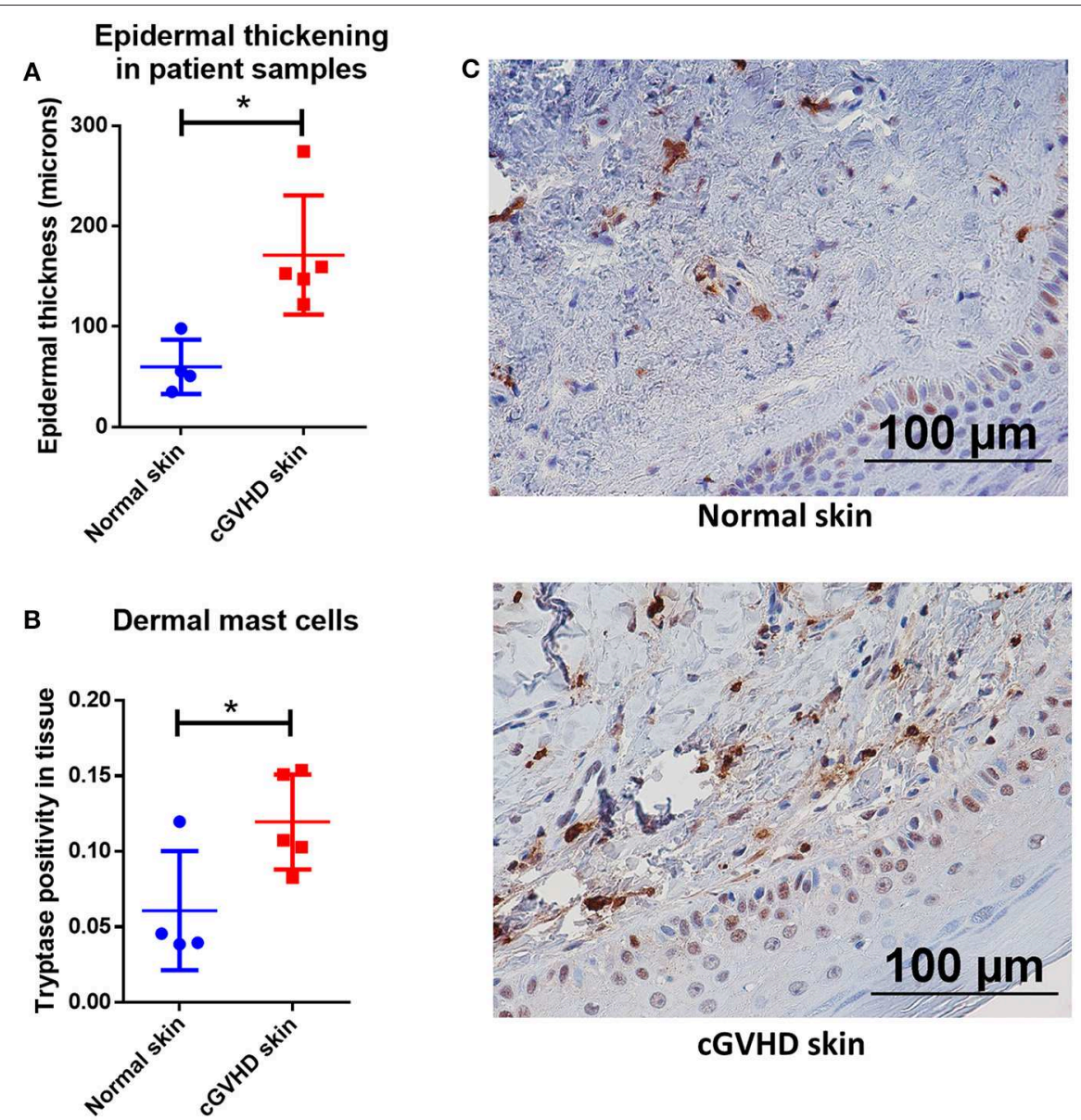

FIGURE 7 | Mast cell numbers are increased in the skin of patients with dermal manifestations of cGVHD. (A) Skin biopsies from patients with cGVHD displayed histologic changes consistent with dermal cGVHD including epidermal thickening as compared to normal controls (normal controls $n=4$, cGVHD patients $n=5$ ). (B) cGVHD patient samples had a significant increase in tryptase staining as measured by Aperio ImageScope in the dermis as compared to normal controls (normal controls $n=4$, cGVHD patients $n=5$ ). (C) Representative images of $(\mathbf{B})$. ${ }^{\star} P=0.01-0.05,{ }^{\star \star} P=0.001-0.01,{ }^{* \star *} P=0.0001-0.001,{ }^{* \star \star \star} P<0.0001, \mathrm{NS}$, not significant.

type. In addition, there is a growing body of literature in human and murine models demonstrating that mast cells and fibroblasts have both direct and indirect interactions, the result of which is increased collagen deposition and fibrosis $(5,38-40)$.

Transplant of WT bone marrow into MC-deficient mice has been previously shown to induce repopulation of mast cells in skin, ear, and other organs within 12 weeks post-HSCT (13). After measuring mast cells by both toluidine blue and avidin staining, we observed that in vivo, mast cells were present in the syngeneic and allo-WT groups at our analysis time, 7 weeks postHSCT. In contrast, almost no mast cells were observed in the allo-MCd group. These findings were confirmed by transcriptlevel analysis of mast cell-specific genes (Figure 3). Therefore, we believe that the mast cells in the allo-WT and syngeneic groups may be recipient derived, although our model does not allow for definitive recipient vs. donor differentiation and there may be other factors driving this disparity in mast cell numbers.

We further saw that the allo-WT animals had significant increases in transcript and protein levels of chemokines
(CCL2, CCL3, CCL4, and CCL5) that can recruit canonical cGVHD effector cells. Levels of these chemokines significantly correlated with our whole-animal GVHD scoring (Figure 4). Additionally, the skin of allo-WT mice had increased tissue damage and a primarily lymphocytic immune infiltrate. We also showed a concomitant increase in T-cell number by immunohistochemistry, alongside data demonstrating increased levels of genes involved in T-cell activation and signaling (Figure 5). However, despite this overactivation, the levels of many T-cell cytokines were shown to not significantly differ between groups in Supplementary Figure 4. We believe this is due to the relatively small difference in number of T-cells alongside the possibility that these cytokines are produced by other cell types, such as macrophages or mast cells.

Importantly, all these changes were ameliorated by mast celldeficiency. Given the mast cell's well-known role as a "sentry cell (41)," it seems that in the context of cGVHD pathogenesis they may be necessary for initial recruitment of inflammatory cells which then cause a feedback loop of further inflammation 
and immune recruitment. Additionally, they may have more direct effects through their impact on fibroblast proliferation. We also show that mast cells are capable of production of many of the chemokines that are upregulated in the allo-WT animals (Figures 6A-C, columns 1, 2, 6).

Ibrutinib is a combined BTK/ITK inhibitor known to be efficacious in treatment of murine and human cGVHD, for which it is currently the only FDA-approved drug $(42,43)$. The Jak1/Jak2 inhibitor, ruxolitinib has been in case studies and is currently in Phase 2 and 3 clinical trials for use in steroidrefractory cGVHD (44-46). Mast cells express many of the signaling molecules targeted by these drugs, and we therefore investigated whether chemokine production would be inhibited when treated with ibrutinib or ruxolitinib $(47,48)$. Indeed, we found a dose-dependent decrease in production of CCL2, CCL3, and CCL4 when treated with ibrutinib and ruxolitinib (Figure 6). This phenomenon was not seen in cells treated with imatinib or fingolimod, which is unsurprising given the lack of exogenous S1P or SCF in the assay (Supplementary Figure 5). Given the overexpression of these same chemokines in the skin of the alloWT vs. allo-MCd mice (Figure 4C) and the reduction in skin symptomology of patients treated with ibrutinib and ruxolitinib (44), it may be that inhibition of mast cell chemokine production is a component of the mechanism by which these inhibitors are clinically efficacious.

Lastly, we show that patients with dermal manifestations of cGVHD have higher levels of mast cells in the skin than normal controls (Figure 7). This data is similar to that seen in our murine model and is suggestive that mast cells may be active in human cGVHD. Further studies directly or indirectly addressing mast cells and correlative biomarkers are warranted as these may assist physicians in diagnosing and predicting skin cGVHD as well as expanding the options for treatment.

The disease pathology of murine models of cGVHD most often takes place within the first 50 days (49). However, mast cells can take as much as 12 weeks to fully reconstitute from the bone marrow to tissue sites (50). This means that in a theoretical model assessing the role of donor-derived mast cells (C57BL/6J or B6.Cg-Kit $\left.{ }^{\mathrm{W}-\mathrm{sh}} / \mathrm{HnihrJaeBsmGllij} \rightarrow \mathrm{LP} / \mathrm{J}\right)$, the cells would not have reconstituted from the bone marrow in time to play a role in disease pathogenesis. Furthermore, restoration of mast cells in $\mathrm{B} 6 . \mathrm{Cg}-\mathrm{Kit}^{\mathrm{W}}{ }^{\mathrm{Sh}} / \mathrm{HnihrJaeBsmGllij}$ by injection of bone-marrow derived mast cells is not feasible in this model, as injected mast cells do not repopulate the skin after they are injected intravenously or intraperitoneally (50). Additionally, neutralizing mast cells using pharmacologic methods or antibody depletion is not feasible. Both of these methods are not sufficiently specific to mast cells and introduce artifacts whose effects would be difficult to deconvolute (51). Specific mast cell depletion is possible in some transgenic models (52), but these often have systemic issues such as Cre activity outside the mast cell lineage (53).

While we believe this study to show exciting insights into the mechanisms of fibrotic cGVHD, it is nonetheless reliant on murine models of cGVHD that do not always recapitulate the human disease. We have demonstrated a novel role for mast cells in murine cGVHD, but its translation to human disease will require further studies. The small number of available banked human samples further hampered our investigation into this phenomenon.

In this current era of targeted therapies, a deeper understanding of disease pathogenesis allows for direct cellular or molecular inhibition. This study is the first in vivo evidence of mast cell involvement in dermal cGVHD. Given the mast cell-dependent nature of this model of cGVHD, and the mast cell's capability to produce many of the same chemokines that we see in the skin of mice with active disease, we propose that mast cell production of chemokines and recruitment of further inflammatory cells may be a critical mechanism of dermal cGVHD pathogenesis. Ibrutinib and ruxolitinib may be capable of modulating this process, and further examination of mast cells in this context may lead to increased therapeutic options for patients who are suffering from cGVHD.

\section{MATERIALS AND METHODS}

\section{Animals}

All animal work was approved by the University of Kentucky Institutional Animal Care and Use Committee and the Department of Laboratory Animal Resources. BALB/cJ, LP/J, C57BL/6J, and B6.Cg-Kit ${ }^{\mathrm{W}-\mathrm{sh}} / \mathrm{HnihrJaeBsmGlliJ}$ mice were all purchased from commercially available sources [Stock numbers 000651 (RRID IMSR_JAX:000651), 000676 (RRID IMSR_JAX:000676), 000664 (RRID IMSR_JAX:000664), and 012861 (RRID MMRRC_012861-UCD), respectively. Jackson Laboratories, Bar Harbor, ME, USA]. All mice were female and were used at 8-10 weeks of age.

\section{Transplant}

C57BL/6J or LP/J mice were sacrificed by $\mathrm{CO} 2$ inhalation, then bone marrow cells and splenocytes were isolated and counted. C57BL/6J and B6.Cg-Kit ${ }^{\mathrm{W}-s h} / \mathrm{HnihrJaeBsmGlliJ}$ recipients were exposed to a single dose of $850 \mathrm{cGy}$ of ionizing radiation from a Cesium source, then injected intravenously with $2.0 \mathrm{e} 6 \mathrm{LP} / \mathrm{J}$ splenocytes and 5.0e6 LP/J bone marrow cells. In the syngeneic group, C57BL/6J mice received the same dose of splenocytes and bone marrow cells from a C57BL/6J mouse. After week 7, blood was collected into heparinized tubes (365965, BD Biosciences, San Jose, CA). Heparin tubes were centrifuged at 1500xG for $10 \mathrm{~min}$, then plasma was harvested and stored at $-80 \mathrm{C}$. Skin, lung, liver, small intestine, colon, kidneys, ear, and tracheal tissue were harvested, trisected, and stored either in formalin for histologic analysis, in RNALater (AM7021, Millipore-Sigma, St. Louis, MO) for RNA stabilization, or snap-frozen over dry ice for protein analysis, then stored at $-80^{\circ} \mathrm{C}$. Spleen was harvested for flow cytometric analysis.

\section{GVHD Scoring}

Recipient mice were monitored daily for survival, and clinical GVHD was assessed weekly by a lab member not directly involved in the planning of the study. Mice were scored for GVHD by assessment of five clinical parameters: weight loss, posture (hunching), activity, fur texture, and skin integrity. Individual mice from coded cages received a score of $0-2$ for each 
criterion (maximum score of 10) that was used as an index of severity and progression of disease (18).

\section{Mast Cell Culture}

Bone marrow was extracted from femurs and tibias isolated from $\mathrm{BALB} / \mathrm{cJ}$ or $\mathrm{C} 57 \mathrm{BL} / 6 \mathrm{~J}$ mice and cultured in DMEM media (12-604F, Lonza, Basel, Switzerland) with 10\% heat-inactivated fetal bovine serum (35-011-CV, Corning Inc., Corning, NY) and $1 \%$ penicillin/streptomycin (120-095-721EA, Quality Biological, Gaithersburg, $\mathrm{MD}$ ) at $37^{\circ} \mathrm{C}, 5 \% \mathrm{CO}_{2}$, in a humidified incubator. After $24 \mathrm{~h}$, the suspension cells were removed and culture in a new flask along with $10 \mathrm{ng} / \mathrm{ml}$ recombinant murine IL-3 (21313, Peprotech, Rocky Hill, NJ). Cells were passaged twice per week, each time leaving behind any adherent cells. After 4-6 weeks, cell phenotype was confirmed by visual morphology and flow cytometry.

\section{Mast Cell Irradiation/Chemotherapy Viability Assay}

Viable mast cells were counted by hemacytometer via trypan blue exclusion and plated at $1 \mathrm{e} 6 / \mathrm{ml}$, then exposed to 8.5 or $20 \mathrm{~Gy}$ single-dose radiation from a cesium source or chemotherapeutic agents. Busulfan and cyclophosphamide were purchased from Cayman Chemicals (14843 and 13849, Ann Arbor, MI) and resuspended in DMSO. Drugging time and conditions were chosen based on clinical usage where cells were drugged with $800 \mathrm{ng} / \mathrm{ml}$ busulfan and/or $30 \mathrm{ug} / \mathrm{ml}$ cyclophosphamide, which corresponded to steady-state serum levels of the drug during clinical utilization (15). Cells were approximately $98 \%$ viable prior to irradiation. Every $24 \mathrm{~h}$, cells were counted and assessed for viability by trypan blue exclusion out to $96 \mathrm{~h}$ post-irradiation or $72 \mathrm{~h}$ post-drugging.

\section{Mast Cell Stimulation and Inhibition Assays} Mast cells were incubated overnight with $100 \mathrm{ng} / \mathrm{ml}$ murine monoclonal anti-DNP IgE, clone SPE-7 (D8406, RRID:AB_259249, Millipore-Sigma, St. Louis, MO) at $37 \mathrm{C}$. The next day, cells were centrifuged and washed twice with fresh media to remove unbound IgE and cells were replated in fresh media with $10 \mathrm{ng} / \mathrm{ml} \mathrm{IL-3.} \mathrm{Stimulation} \mathrm{was} \mathrm{to} \mathrm{the} \mathrm{relevant}$ wells added in the form of $10 \mathrm{ng} / \mathrm{ml} \mathrm{IL-33} \mathrm{and/or} 100 \mathrm{ng} / \mathrm{ml}$ DNP-BSA for $24 \mathrm{~h}$ at $37 \mathrm{C}$, then cells were centrifuged and supernatant was collected. For the drug inhibition assays, ibrutinib, ruxolitinib, imatinib, and fingolimod were purchased from Cayman Chemical (936563-96-1, 941678-49-5, 22012757-1, 162359-55-9, Ann Arbor, MI) and reconstituted per the manufacturer's instructions. Drug stocks were diluted in media and incubated with mast cells after the overnight IgE incubation step. Cells were treated for $1 \mathrm{~h}$ at $37 \mathrm{C}$, then washed out with fresh media twice. Cells were then stimulated as per above.

\section{Degranulation by CD107a Expression}

Cells were incubated at $1 \mathrm{e} 6 / \mathrm{ml}$ with $\mathrm{IgE}$ overnight then washed and plated in new media, as above. $0.66 \mu \mathrm{l} / \mathrm{ml}$ Golgistop (554724, BD Biosciences, San Jose, CA) was added along with AF594-CD107a antibody, clone 1D4B (121624, RRID:AB_2616690, Biolegend, San Diego, CA), then cells were stimulated with $100 \mathrm{ng} / \mathrm{ml}$ DNP-BSA and/or $50 \mathrm{ng} / \mathrm{ml} \mathrm{SCF}$ (25003, Peprotech) for $4 \mathrm{~h}$ at $37 \mathrm{C}$. Cells were then collected, washed, and fixed/permeabilized with Fix/Perm reagent (554714, BD Biosciences). Cells were then stained with BV421-CD117 (clone 2B8, 105827, RRID:AB_10898120) and PE-FceR1a (clone MAR1, 134307, RRID:AB_1626104) (Biolegend) in perm/wash buffer for $20 \mathrm{~min}$ at $4 \mathrm{C}$, washed, and analyzed by flow cytometry for CD117/FceR1a ${ }^{+/+} \mathrm{CD}_{107^{+/-}}$expression.

\section{Histology}

Organs were collected from mice and suspended in $4 \%$ formalin for $48 \mathrm{~h}$, then $70 \%$ ethanol for 7 days. After this, samples were cut into slices, processed, and embedded in paraffin blocks. All sections shown were sectioned at 5 micron thickness. Masson's trichrome staining was performed according to manufacturer protocol (26367-Series, Electron Microscopy Sciences, Hatfield, PA). $\mathrm{H}+\mathrm{E}$ stains were performed by the University of Kentucky Surgical Pathology core and assessed by a blinded, board-certified pathologist according to a previously published organ-specific GVHD scoring system $(18,54)$. Skin slides were stained with Toluidine Blue O (364-12, Millipore Sigma) and assessed as toluidine blue-positive cells per high-power field. Counts were verified by a separate, blinded researcher. Texas Red-conjugated avidin was purchased from Rockland Immunochemicals (A00309, Limerick, PA) and resuspended at $2 \mathrm{mg} / \mathrm{ml}$ in deionized water. This was diluted 1:500 and sections were stained for $1 \mathrm{~h}$, washed 3 times in PBS, dehydrated to xylene, and mounted with Fluoroshield Mounting Medium with DAPI (ab104139, Abcam, Boston, MA).

\section{Immunohistochemistry}

Slides were baked at $80^{\circ} \mathrm{C}$ for $15 \mathrm{~min}$, then rehydrated through xylene, 100,95 , and $70 \%$ ethanol into $\mathrm{H}_{2} \mathrm{O}$. Antigen retrieval was performed in citrate Target Retrieval Solution (S169984-2, Agilent, Santa Clara, CA), pH 6.0, in a pressure cooker. Slides were blocked for $30 \mathrm{~min}$ in PowerBlock (HK083-50K, Biogenex, Fremont, CA), then incubated with primary antibody at a 1:200 dilution in PowerBlock. Antibodies used were rabbit anti-mouse CD3e, clone D4V8L (99940, RRID:AB_2755035, Cell Signaling Technologies, Danvers, MA), and mouse anti-human tryptase, clone AA1 (ab2378, RRID:AB_303023, Abcam, Boston, MA). Slides were washed in PBS $+0.1 \%$ Tween 20 (PBS-T) and then incubated with secondary antibody. For CD3 staining, a drop of Dako Envision+ HRP Anti-Rabbit Polymer (K4003, RRID:AB_2630375, Agilent) was added to slides for $1 \mathrm{~h}$, then washed with PBS-T. For tryptase staining, Goat Anti-Mouse Immunoglobulin/HRP (P0260, RRID:AB_2636929, Agilent) was used at a 1:1 dilution for $10 \mathrm{~min}$ and then washed with PBS-T. After washing, DAB chromogen (ab64238, Abcam) was prepared according to manufacturer's instructions and added to slides while observing the staining under a microscope for $30 \mathrm{~s}$ (tryptase staining) or $2 \mathrm{~min}$ (CD3 staining). The reaction was quenched in $\mathrm{diH}_{2} \mathrm{O}$, then dehydrated to xylene. Slides were mounted using Richard-Allan Scientific mounting medium (4112APG, Thermo Fisher Scientific, Waltham, MA). 


\section{Microscopy and Analysis}

Histology slides were imaged by Aperio Scanscope XT digital slide scanner or by a color camera system attached to a Nikon Eclipse $80 \mathrm{i}$ microscope. All scale bars are $100 \mu \mathrm{m}$. Trichrome and CD3 IHC slides were digitally scanned by an Aperio ScanScope XT system. Dermal thickness was measured with the ruler tool across 10-20 areas of tissue. Aperio methodology and results were checked and verified by CDJ. CD3 and tryptase positivity was measured across the whole tissue by the Aperio Positive Pixel Count V9 algorithm. Counts were also verified by ImageJ IHC profiler analysis by a blinded, independent researcher, RK. Fluorescence images were acquired on a Nikon Eclipse TE2000-U microscope equipped with a DS-Qi1Mc camera.

\section{Flow Cytometry}

Splenocytes were phenotyped using two different antibody panels. The first panel consisted of lymphocyte and T-cell stains and allowed for identification of CD45, CD3, CD4, CD8, and FoxP3 subsets. The second panel identified B-cells, neutrophils, macrophages, and dendritic cells using stains for CD19, CD11c, CD11b, F4/80, and Ly6G (Gating scheme for both panels in Supplementary Figure 6). Splenic tissue was forced through a 70 micron filter to create a single cell suspension and cells were washed with PBS containing 4\% FBS and incubated with FcR-block anti-mouse CD16/CD32 (14-016182, RRID:AB_467133, Clone 93, eBioscience, San Diego, CA). Cells then were incubated in a pre-optimized concentration of antibodies in a total volume of $100 \mu \mathrm{L}$. For intracellular staining for FoxP3, fixation and permeabilization were done as per protocol using the fixation/permeabilization concentrate and diluent (00-5123-43, eBioscience). The following antibody clones were used for splenocyte characterization:

FITC-CD3 (REA641, 130-119-798, RRID:AB_2751851), APC-CD4 (GK1.5, 130-091-611, RRID:AB_871692), APCVio770-CD45 (30F11, 130-118-687, RRID:AB_2733121), APC-CD11c (N418, 130-091-844, RRID:AB_244272), PE-CD19 (6D5, 130-092-041, RRID:AB_871638), FITC-F4/80 (REA126, 130-102-988, RRID:AB_2651700) (Miltenyi Biotec, Bergisch Gladbach, Germany).

PerCP-Cy5.5-CD11b (M1/70, 101228, RRID:AB_893232), PerCP-Cy5.5-CD8 (53-6.7, 100733, RRID:AB_2075239) (Biolegend, San Jose, CA).

PE-Cy7-Ly6G (RB6-8C5, 25-5931-81, RRID:AB_469662), APC-eFluor780-MHCII (M5/114.15.2, 47-5321-82, RRID:AB_1548783), PE-FoxP3 (FJK-16s, 12-5773-80, RRID:AB_465935) (eBioscience).

Mast cells were phenotyped using the following antibody clones:

BV421-CD117 (clone 2B8, 105827, RRID:AB_10898120), PEFceR1a (clone MAR-1, 134307, RRID:AB_1626104) (Biolegend)

All cells were analyzed on a BD LSR II cytometer (BD Biosciences) using single-stained OneComp eBeads (01-111141, Thermo Fisher Scientific, Waltham, MA) to determine compensation values. All gates were set using fluorescenceminus-one (FMO) controls. Flow cytometry data were analyzed using FlowJo V10 software (FlowJo Inc., Ashland, OR).

\section{RNA Isolation and NanoString Analysis}

Tissue was frozen in RNALater as described in "Transplant" methods. The tissue was thawed, then suspended in $1 \mathrm{ml}$ of Ribozol RNA isolation solution (VWR, Radnor, PA), and homogenized using a Tissuemiser homogenizer (Thermo Fisher, Waltham, MA). RNA was isolated from the homogenate per the Ribozol manufacturer's instructions. RNA was quantitated using the Qubit RNA BR assay kit in the Qubit Fluorometer (Thermo Fisher). Hundred nanogram of RNA was loaded into the cartridge of the NanoString Myeloid Innate Immunity panel and run on the nCounter Flex system at the University of Kentucky Clinical Genomics core per the manufacturer's instructions (NanoString Technologies, Seattle, WA). Data normalization and analysis was performed in the nSolver software.

\section{Real-Time Quantitative PCR}

For cDNA synthesis and qPCR, RNA was quantified using a Nanodrop instrument (Thermo Fisher), and 100 ng was loaded into the iScript cDNA Synthesis Kit (1708891, Bio-Rad, Hercules, CA). For IL21 gene analysis, a predesigned primer probe mix was purchased from Integrated DNA Technologies (Assay ID: Mm.PT.58.7853071, Integrated DNA Technologies, San Jose, $\mathrm{CA}$ ) and normalized using ACTB as a housekeeping gene (Assay ID: Mm.PT.39a.22214843.g). cDNA was assayed using the Luna Universal Probe qPCR master-mix (M3004X, New England Biolabs, Ipswich, MA), on an Applied Biosystems StepOne Real-Time PCR System (Applied Biosystems, Foster City, CA). Relative quantitation was performed using the delta-delta CT method, described previously (55).

\section{Tissue Lysate Preparation}

Tissue was snap-frozen on dry ice as described in the "Transplant" methods section. The tissue was thawed, then suspended in $500 \mathrm{ml} 1 \times$ RIPA buffer (89900, Thermo Fisher, Waltham, MA) with protease inhibitors added (cOmplete Protease Inhibitor Cocktail, 4693159001, Roche, Basel, Switzerland). Tissue was homogenized using a Tissuemiser homogenizer (Thermo Fisher). Homogenate was vortexed for $30 \mathrm{~s}$, then put on ice for $10 \mathrm{~min}$. This procedure was repeated 3 times, then homogenates were spun down at 14,000 $\times \mathrm{G}$ for $10 \mathrm{~min}$ at $4 \mathrm{C}$. Supernatant was moved to a new tube and quantified by Pierce BCA protein assay kit (23225, Thermo Fisher).

\section{Measurement of Cytokines and \\ Chemokines}

The levels of TNF- $\alpha$, IL-6, IFN- $\gamma$, IL-2, IL-4, IL-10, and IL- $17 \alpha$ were determined in plasma samples through the Mouse Th1/Th2/Th17 Cytometric Bead Array kit (560485, BD Biosciences, San Jose, CA). Bead fluorescence was measured using the BD Biosciences LSRII flow cytometer and analyzed with the accompanying software. Protein levels of CCL5, CCL11, CCL17, CXCL1, CXCL9, CXCL10, CXCL13, CXCL5, CCL22, CCL2, CCL3, and CCL4 in tissue and mast cell supernatants were analyzed with the LEGENDplex Mouse Proinflammatory Chemokine Panel (740007, Biolegend) per the manufacturer's instructions. Supernatants were loaded without 
dilution. Hundred micrograms of tissue lysate were loaded for analysis of chemokine levels in the skin, although final values displayed in the paper were normalized to nalyseds per milliliter per microgram of protein loaded. Bead fluorescence was measured using the BD Biosciences LSRII flow cytometer and analyzed in the Biolegend LEGENDplex data analysis software.

\section{Fibroblast Proliferation Assay}

Fibroblasts were cultured from the skin of C57BL/6J mice as previously described (56). After 3 weeks of culture, cells were nearly confluent in a $10 \mathrm{~cm}$ petri dish. Media was aspirated off and $3 \mathrm{~mm}$ of trypsin was added to the dish for $5 \mathrm{~min}$. After $5 \mathrm{~min}$, cells were scraped off using a sterile scraper and spun down at $350 \times \mathrm{G}$ for $5 \mathrm{~min}$. Cells were resuspended in $6 \mathrm{ml}$ and plated equally into a 6 well plate in DMEM $+10 \%$ FBS $+1 \%$ penicillin/streptomycin. After $4 \mathrm{~h}$ to allow the cells to adhere, cells were counted on an inverted microscope across 4 fields of view per well at 200x magnification. At this point, $1 \mathrm{ml}$ of mast cell supernatants or fresh media was added to the $1 \mathrm{ml}$ of culture in each well. Cells were counted again $24 \mathrm{~h}$ later, and again after $48 \mathrm{~h}$.

\section{Human Tissue Samples}

All GVHD samples were from patients at the University of Kentucky Markey Cancer Center who had undergone an allo-HSCT with a subsequent diagnosis of chronic GVHD. Chart review and sample acquisition was performed by the honest broker of the University of Kentucky Biospecimen Procurement and Translational Pathology Core and samples were sectioned from paraffin-embedded tissue. All normal and cGVHD skin samples were derived from surgical specimens left over from clinical care that were banked by the UK Biospecimen Procurement Core under a waiver of consent from the UK IRB. All samples were deidentified and were not able to be linked to patient records by any investigators involved in the study. As a result of this, the University of Kentucky Institutional Review Board deemed that these samples did not meet the Department of Health and Human Services (DHHS) definition of human subjects or the Food \& Drug Administration's (FDA) definition of human subjects.

\section{Figure Preparation and Statistics}

All figures were prepared in GraphPad Prism 6 and 7 (GraphPad, San Diego, CA), FlowJo V10 (FlowJo Inc., Ashland, OR), or NanoString nSolver (NanoString Technologies, Seattle, WA) software packages. Experimental data are expressed as means \pm standard deviation unless noted otherwise in the figure legend. Differences between two groups were analyzed by the two-tailed independent $t$-test. Differences between three or more groups were analyzed by one-way ANOVA, with a Tukey's post-hoc multiple comparisons test to determine the significance between groups. $P-\leq 0.05$ were considered statistically significant. ${ }^{*} P$ $=0.01-0.05,{ }^{* *} P=0.001-0.01,{ }^{* * *} P=0.0001-0.001,{ }^{* * * *} P<$ 0.0001 , NS, not significant.

\section{DATA AVAILABILITY STATEMENT}

Nanostring data is stored in the publicly available NCBI Gene Expression Omnibus database (accession number GSE128704).
Other data in this study is available from the corresponding author upon request.

\section{ETHICS STATEMENT}

The animal study was reviewed and approved by University of Kentucky Institutional Animal Care and Use Committee. All studies involving anonymized clinical samples were considered by the University of Kentucky Institutional Review Board to be non-human research, as the studies did not meet the Department of Health and Human Services (DHHS) definition of human subjects or the Food \& Drug Administration's (FDA) definition of human subjects.

\section{AUTHOR CONTRIBUTIONS}

ES and GH wrote the manuscript. ES, SP, JS, and GH designed the experiments. ES, SP, RK, and TH performed the experiments and analyzed the data. NH, JD, MK, and CJ provided blinded pathological and phenotypic analysis.

\section{FUNDING}

This study was supported via Investigator Support Funds from the University of Kentucky/Markey Cancer Center awarded to $\mathrm{GH}$.

\section{ACKNOWLEDGMENTS}

This research was supported by the following core labs: Flow Cytometry and Cell Sorting Shared Resource Facility, Biospecimen Procurement and Translational Pathology Shared Resource Facility, and the Oncogenomics Shared Resource Facility, all through the University of Kentucky Markey Cancer Center (P30CA177558). Research reported in this publication was also supported by an Institutional Development Award (IDeA) from the National Institute of General Medical Sciences of the National Institutes of Health under grant number P20GM103527, and Aperio services were supported through the NIA/NIH ADC P30 AGO28383.

\section{SUPPLEMENTARY MATERIAL}

The Supplementary Material for this article can be found online at: https://www.frontiersin.org/articles/10.3389/fimmu. 2019.02470/full\#supplementary-material

Supplementary Figure 1 | Pathology is not significantly different after allogeneic transplant between allo-WT and allo-MCd in lung, small intestine, colon, or liver. Pathology score is unchanged between all groups in (A) lung, (B) small intestine, (C) colon, or (D) liver. Scoring was performed as described previously $(18,54)$ by a blinded pathologist. Syngeneic $(n=6)$, allo-WT $(n=16)$, and allo-MCd $(n=14)$. Data is combined from two independent transplants. ${ }^{*} P=0.01-0.05,{ }^{* *} P=$ $0.001-0.01,{ }^{* * *} P=0.0001-0.001,{ }^{* * * *} P<0.0001$, NS, not significant.

Supplementary Figure 2 | Mast cells are present in multiple tissues after transplant and display evident degranulation in allo-WT mice. (A) Mast cell cite counts and toluidine blue-stained representative images in the trachea of syngeneic $(n=6)$, allo-WT $(n=16)$, and allo-MCd $(n=14)$ animals. Mast cell counts per high-power field (400x) are displayed in the first column alongside images from animals whose mast cell count was nearest the mean value for the 
group. Mast cells (arrows) were denoted by metachromatic staining and granular appearance. (B) Skin and ear sections were stained with avidin and mast cells counted per high-power field (blue = DAPI, red = avidin). (C) Representative images of avidin-stained mast cells in the ear. (D) Degranulation was evident in this representative image of skin from allo-WT mice. ${ }^{*} P=0.01-0.05,{ }^{* *} P=$ $0.001-0.01,{ }^{* * *} P=0.0001-0.001,{ }^{* * * *} P<0.0001, \mathrm{NS}$, not significant

Supplementary Figure $\mathbf{3}$ | Markers of many immune subsets in the spleen and skin are not significantly changed. (A) Myeloid subsets are unchanged in the spleen 7 weeks after allogeneic transplant. MHCII/CD11 $\mathrm{C}^{+/+}$dendritic cells, Ly6G+ neutrophils, or CD11b/F4/80+/+ macrophages have no significant differences in proportion or overall count (data not shown) in the spleen after induction of cGVHD. (B) There were no significant differences in splenic proportion or count (data not shown) of the lymphoid subsets analyzed (CD45+ lymphocytes, CD45/CD19 ${ }^{+/+}$B-cells, CD45/CD3 ${ }^{+/+}$T-cells, CD45/CD3/CD4/FoxP3+/+/+/+ T-regulatory cells). This implies that the dermal cGVHD symptomology evident in these mice is driven more strongly by local factors than purely by increased alloreactivity, a conclusion which is consistent with many theories regarding the pathogenesis of fibrotic cGVHD. (C) There is no significant difference in the skin in CD19 transcript (measured by qPCR) or eosinophil/neutrophil counts (counted by a pathologist by $\mathrm{H}+\mathrm{E}$ morphology). ${ }^{*} P=0.01-0.05,{ }^{* *} P=0.001-0.01,{ }^{* * *} P=$ $0.0001-0.001,{ }^{* * * *} P<0.0001$, NS, not significant.

Supplementary Figure 4 | Pathogenic cytokines are expressed at low levels in the skin and are largely unchanged between groups. (A) PANTHER pathway analysis demonstrating an increase in genes related to "Inflammation mediated by chemokine and cytokine signaling" in allo-WT relative to allo-MCd. (B) Heatmap analysis and selected genes showing lowered expression of cytokine signaling

\section{REFERENCES}

1. Copelan EA. Hematopoietic stem-cell transplantation. N Engl J Med. (2006) 354:1813-26. doi: 10.1056/NEJMra052638

2. Arai S, Arora M, Wang T, Spellman SR, He W, Couriel DR, et al. increasing incidence of chronic graft-vs.-host disease in allogeneic transplantation a report from CIBMTR. Biol Blood Marrow Transplant. (2015) 21:26674. doi: 10.1016/j.bbmt.2014.10.021

3. Flowers MED, Martin PJ. How we treat chronic graft-vs.-host disease. Blood. (2015) 125:606-15. doi: 10.1182/blood-2014-08-551994

4. Galli SJ, Tsai M. Mast cells in allergy and infection: versatile effector and regulatory cells in innate and acquired immunity. Eur J Immunol. (2010) 40:1843-51. doi: 10.1002/eji.201040559

5. Pincha N, Hajam EY, Badarinath K, Batta SPR, Masudi T, Dey R, et al. PAI1 mediates fibroblast-mast cell interactions in skin fibrosis. J Clin Invest. (2018) 128:1807-19. doi: 10.1172/JCI99088

6. Hügle T. Beyond allergy: the role of mast cells in fibrosis. Swiss Med Wkly. (2014) 144:w13999. doi: 10.4414/smw.2014.13999

7. Veerappan A, O'Connor NJ, Brazin J, Reid AC, Jung A, McGee D, et al. Mast cells: a pivotal role in pulmonary fibrosis. DNA Cell Biol. (2013) 32:20618. doi: 10.1089/dna.2013.2005

8. Claman HN, Choi KL, Sujansky W, Vatter AE. Mast cell 'disappearance' in chronic murine graft-vs-host disease (GVHD)-ultrastructural demonstration of 'phantom mast cells'. J Immunol. (1986) 137:2009-13.

9. Claman HN. Mast cell depletion in murine chronic graft-vs.-host disease. $J$ Invest Dermatol. (1985) 84:246-8. doi: 10.1111/1523-1747.ep12265302

10. Levi-Schaffer F, Mekori YA, Segal V, Claman HN. Histamine release from mouse and rat mast cells cultured with supernatants from chronic murine graft-vs.-host splenocytes. Cell Immunol. (1990) 127:146-58. doi: 10.1016/0008-8749(90)90121-7

11. Claman HN. Mast cells, T cells and abnormal fibrosis. Immunol Today. (1985) 6:192-5. doi: 10.1016/0167-5699(85)90115-X

12. Levi-Schaffer F, Segal V, Baram D, Mekori YA. Effect of coculture of rodent mast cells with murine chronic graft-vs.-host disease (cGVHD)-derived fibroblasts. J Allergy Clin Immunol. (1992) 89:501-9. doi: 10.1016/0091-6749(92)90316-T

13. Grimbaldeston MA, Chen CC, Piliponsky AM, Tsai M, Tam SY, Galli SJ. Mast cell-deficient W-sash c-kit mutant KitW-sh/W-sh mice as a model genes in allo-MCd animals compared to allo-WT animals as measured by NanoString. Heatmaps and gene pathway annotations were generated using NanoString nSolver software. (C) Protein levels were measured in the skin for IL-6, TNF-alpha, IL-4, and IFN-gamma. (D) Protein levels in plasma (syngeneic $n=3$, allo-WT $n=8$, and allo-MCd $n=7$ ). ${ }^{*} P=0.01-0.05,{ }^{* *} P=0.001-0.01,{ }^{* * *} P=$ $0.0001-0.001,{ }^{* * * *} P<0.0001$, NS, not significant.

Supplementary Figure $\mathbf{5}$ | Chemokine production is not reduced after treatment with imatinib or fingolimod and cell viability is unaffected by drugging. Mast cells produce high levels of (A) CCL2, (B) CCL3, and (C) CCL4 upon stimulation with $\lg \mathrm{E}+$ antigen or IgE + antigen $+\mathrm{IL}-33$ (column 1 vs. columns 2 and 6).

Production of these chemokines is not decreased by treatment with either imatinib or fingolimod. Results shown are representative of 2-4 independent assays. Error bars are the $S D$ of technical replicates. Chemokine assays were performed using the LEGENDplex Inflammatory Chemokine Assay kit, which measures levels of 13 chemokines. Mast cells did not produce significant amounts of CCL5, CCL11, CCL17, CXCL1, CXCL9, CXCL10, CXCL13, CXCL5, or CCL22 (data not shown). (D) Mast cell viability was unaffected after $24 \mathrm{~h}$ of drugging with either imatinib, fingolimod, ibrutinib, or ruxolitinib. ${ }^{*} P=0.01-0.05,{ }^{* *} P=0.001-0.01,{ }^{* * *} P=$ $0.0001-0.001,{ }^{* * * *} P<0.0001$, NS, not significant.

Supplementary Figure 6 | Flow cytometry gating schemes. Gating schema for flow cytometry panels run on spleen (Supplementary Figure 3). (A) Gating scheme for a panel to assay T-cell subsets in the spleen. (B) Gating scheme for a panel to assay myeloid subsets and B-cells in the spleen. Red samples are fully stained, while blue, or orange are FMO controls. ${ }^{*} P=0.01-0.05,{ }^{*} P=$ $0.001-0.01,{ }^{* * *} P=0.0001-0.001,{ }^{* * * *} P<0.0001$, NS, not significant.

for investigating mast cell biology in vivo. Am J Pathol. (2005) 167:83548. doi: 10.1016/S0002-9440(10)62055-X

14. Soule BP, Brown JM, Kushnir-Sukhov NM, Simone NL, Mitchell JB, Metcalfe DD. Effects of gamma radiation on FceRI and TLR-mediated mast cell activation. J Immunol. (2007) 179:32763286. doi: 10.4049/jimmunol.179.5.3276

15. Tutschka PJ, Copelan EA, Klein JP. Bone marrow transplantation for leukemia following a new busulfan and cyclophosphamide regimen. Blood. (1987) 70:1382-8.

16. Yeh RF, Pawlikowski MA, Blough DK, McDonald GB, O’Donnell PV, Rezvani A, et al. Accurate targeting of daily intravenous busulfan with 8-hour blood sampling in hospitalized adult hematopoietic cell transplant recipients. Biol Blood Marrow Transpl J Am Soc Blood Marrow Transplant. (2012) 18:265-72. doi: 10.1016/j.bbmt.2011.06.013

17. Hamilton BL, Parkman R. Acute and chronic graft-vs.-host disease induced by minor histocompatibility antigens in mice. Transplantation. (1983) 36:1505. doi: 10.1097/00007890-198308000-00008

18. Cooke KR, Kobzik L, Martin TR, Brewer J, Delmonte J, Crawford JM., et al. An experimental model of idiopathic pneumonia syndrome after bone marrow transplantation: I. The roles of minor $\mathrm{H}$ antigens and endotoxin. Blood. (1996) 88:3230-9.

19. Martires KJ, Baird K, Steinberg SM, Grkovic L, Joe GO, Williams KM, et al. Sclerotic-type chronic GVHD of the skin: clinical risk factors, laboratory markers, and burden of disease. Blood. (2011) 118:42507. doi: 10.1182/blood-2011-04-350249

20. Shulman HM, Kleiner D, Lee SJ, Morton T, Pavletic SZ, Farmer E, et al. Histopathologic diagnosis of chronic graft vs. host disease. Biol. Blood Marrow Transplant. (2015) 21:589-603. doi: 10.1016/j.bbmt.2005. 10.023

21. Taylor DK, Mittereder N, Kuta E, Delaney T, Burwell T, Dacosta K, et al. T follicular helper-like cells contribute to skin fibrosis. Sci Transl Med. (2018) 10:eaaf5307. doi: 10.1126/scitranslmed.aaf5307

22. Pohlers D, Brenmoehl J, Löffler I, Müller CK, Leipner C, Schultze-Mosgau $S$, et al. TGF- $\beta$ and fibrosis in different organs - molecular pathway imprints. Biochim Biophys Acta BBA - Mol Basis Dis. (2009) 1792:74656. doi: 10.1016/j.bbadis.2009.06.004

23. Wynn TA. Cellular and molecular mechanisms of fibrosis. J Pathol. (2008) 214:199-210. doi: 10.1002/path.2277 
24. Bergstresser PR, Tigelaar RE, Tharp MD. Conjugated avidin identifies cutaneous rodent and human mast cells. J Invest Dermatol. (1984) 83:2148. doi: 10.1111/1523-1747.ep12263584

25. Zhang T, Liu R, Che D, Pundir P, Wang N, Han S, et al. A mast cell-specific receptor is critical for granuloma induced by intrathecal morphine infusion. $J$ Immunol. (2019) 203:ji1801423. doi: 10.4049/jimmunol.1801423

26. Mi H, Huang X, Muruganujan A, Tang H, Mills C, Kang D, et al. PANTHER version 11: expanded annotation data from Gene Ontology and Reactome pathways, and data analysis tool enhancements. Nucleic Acids Res. (2017) 45:D183-9. doi: 10.1093/nar/gkw1138

27. Luther SA, Cyster JG. Chemokines as regulators of $\mathrm{T}$ cell differentiation. Nat Immunol. (2001) 2:102-7. doi: 10.1038/84205

28. Patterson SJ, Pesenacker AM, Wang AY, Gillies J, Mojibian M, Morishita $\mathrm{K}$, et al. $\mathrm{T}$ regulatory cell chemokine production mediates pathogenic $\mathrm{T}$ cell attraction and suppression. J Clin Invest. (2016) 126:1039-51. doi: 10.1172/JCI83987

29. Mukai K, Tsai M, Saito H, Galli SJ. Mast cells as sources of cytokines, chemokines and growth factors. Immunol Rev. (2018) 282:121-50. doi: 10.1111/imr.12634

30. Choi SW, Hildebrandt GC, Olkiewicz KM, Hanauer DA, Chaudhary $\mathrm{MN}$, Silva IA, et al. CCR1/CCL5 (RANTES) receptor-ligand interactions modulate allogeneic T-cell responses and graft-vs.host disease following stem-cell transplantation. Blood. (2007) 110:3447-55. doi: 10.1182/blood-2007-05-087403

31. Blirando K, Milliat F, Martelly I, Sabourin JC, Benderitter M, François A. Mast cells are an essential component of human radiation proctitis and contribute to experimental colorectal damage in mice. Am J Pathol. (2011) 178:640-51. doi: 10.1016/j.ajpath.2010.10.003

32. Hall AG, Tilby MJ. Mechanisms of action of, and modes of resistance to, alkylating agents used in the treatment of haematological malignancies. Blood Rev. (1992) 6:163-73. doi: 10.1016/0268-960X(92)90028-O

33. Schuurhuis GJ, Meel MH, Wouters F, Min LA, Terwijn M, de Jonge NA, et al. Normal hematopoietic stem cells within the AML bone marrow have a distinct and higher ALDH activity level than co-existing leukemic stem cells. PLoS ONE. (2013) 8:e78897. doi: 10.1371/journal.pone.0078897

34. Marino A, Sakamoto T, Robador PA, Tomita K, Levi R. S1P receptor 1mediated anti-renin-angiotensin system cardioprotection: pivotal role of mast cell aldehyde dehydrogenase type 2. J. Pharmacol Exp Ther. (2017) 362:230-42. doi: 10.1124/jpet.117.241976

35. Qu Z, Liebler JM, Powers MR, Galey T, Ahmadi P, Huang XN, et al. Mast cells are a major source of basic fibroblast growth factor in chronic inflammation and cutaneous hemangioma. Am J Pathol. (1995) 147:564-73.

36. Leveson-Gower DB, Sega EI, Kalesnikoff J, Florek M, Pan Y, Pierini A, et al. Mast cells suppress murine GVHD in a mechanism independent of CD4+ CD25+ regulatory $\mathrm{T}$ cells. Blood. (2013) 122:3659-65. doi: 10.1182/blood-2013-08-519157

37. Blazar B, White ES, Couriel D. Understanding Chronic GVHD from Different Angles. Biol Blood Marrow Transplant. (2012) 18:S184-8. doi: 10.1016/j.bbmt.2011.10.025

38. Abel M, Vliagoftis H. Mast cell-fibroblast interactions induce matrix metalloproteinase-9 release from fibroblasts: role for IgE-mediated mast cell activation. J Immunol. (2008) 180:3543-50. doi: 10.4049/jimmunol.180.5.3543

39. Arbi S, Eksteen EC, Oberholzer HM, Taute H, Bester MJ. Premature collagen fibril formation, fibroblast-mast cell interactions and mast cellmediated phagocytosis of collagen in keloids. Ultrastruct Pathol. (2015) 39:95103. doi: $10.3109 / 01913123.2014 .981326$

40. Garbuzenko E, Nagler A, Pickholtz D, Gillery P, Reich R, Maquart FX, et al. Human mast cells stimulate fibroblast proliferation, collagen synthesis and lattice contraction: a direct role for mast cells in skin fibrosis. Clin Exp Allergy. (2002) 32:237-46. doi: 10.1046/j.1365-2222.2002.01293.x

41. Hellman LT, Akula S, Thorpe M, Fu Z. Tracing the origins of IgE, mast cells, and allergies by studies of wild animals. Front Immunol. (2017) 8:1749. doi: 10.3389/fimmu.2017.01749

42. Dubovsky JA, Flynn R, Du J, Harrington BK, Zhong Y, Kaffenberger B, et al. Ibrutinib treatment ameliorates murine chronic graft-vs.-host disease. J Clin Invest. (2014) 124:4867-76. doi: 10.1172/JCI75328

43. Miklos D, Cutler CS, Arora M, Waller EK, Jagasia M, Pusic I, et al. Ibrutinib for chronic graft-vs.-host disease after failure of prior therapy. Blood. (2017) 130:2243-50. doi: 10.1182/blood-2017-07-793786
44. Sarmiento Maldonado M, Ramírez Villanueva P, Bertín Cortes-Monroy P, Jara Arias V, Soto Donoso K, Uribe Gonzalez P, et al. Compassionate use of ruxolitinib in acute and chronic graft vs. host disease refractory both to corticosteroids and extracorporeal photopheresis. Exp Hematol Oncol. (2017) 6:32. doi: 10.1186/s40164-017-0092-3

45. Study of Ruxolitinib in Sclerotic Chronic Graft-vs.-Host Disease After Failure of Systemic Glucocorticoids - Full Text View - ClinicalTrials.gov. Available online at: https://clinicaltrials.gov/ct2/show/NCT03616184 (accessed March 14, 2019).

46. A Study of Ruxolitinib vs. Best Available Therapy (BAT) in Patients With Steroid-refractory Chronic Graft vs. Host Disease (GvHD) After Bone Marrow Transplantation (REACH3) - Full Text View - ClinicalTrials.gov. Available online at: https://clinicaltrials.gov/ct2/show/NCT03112603 (accessed March 14, 2019).

47. Morales JK, Falanga YT, Depcrynski A, Fernando J, Ryan JJ. Mast cell homeostasis and the JAK-STAT pathway. Genes Immun. (2010) 11:599608. doi: 10.1038/gene.2010.35

48. Huang W, Morales JL, Gazivoda VP, August A. Nonreceptor tyrosine kinases ITK and BTK negatively regulate mast cell proinflammatory responses to lipopolysaccharide. J Allergy Clin Immunol. (2016) 137:1197-205. doi: 10.1016/j.jaci.2015.08.05

49. Schroeder MA, DiPersio JF. Mouse models of graft-vs.-host disease: advances and limitations. Dis Model Mech. (2011) 4:318-33. doi: 10.1242/dmm.006668

50. Wolters PJ, Mallen-St Clair J, Lewis CC, Villalta SA, Baluk P, Erle DJ, et al. Tissue-selective mast cell reconstitution and differential lung gene expression in mast cell-deficient Kit(W-sh)/Kit(W-sh) sash mice. Clin Exp Allergy. (2005) 35:82-8. doi: 10.1111/j.1365-2222.2005.02136.x

51. Jönsson F, Daëron M. Mast cells and company. Front Immunol. (2012) 3:16. doi: 10.3389/fimmu.2012.00016

52. Youngblood BA, Brock EC, Leung J, Falahati R, Bochner BS, Rasmussen HS, et al. Siglec- 8 antibody reduces eosinophil and mast cell infiltration in a transgenic mouse model of eosinophilic gastroenteritis. JCI Insight. (2019) 4:126219. doi: 10.1172/jci.insight.126219

53. Cildir G, Pant H, Lopez AF, Tergaonkar V. The transcriptional program, functional heterogeneity, and clinical targeting of mast cells. J Exp Med. (2017) 214:2491-506. doi: 10.1084/jem.20170910

54. Kaplan DH, Anderson BE, McNiff JM, Jain D, Shlomchik MJ, Shlomchik WD. Target antigens determine graft-vs.-host disease phenotype. J Immunol. (2004) 173:5467-75. doi: 10.4049/jimmunol.173.9.5467

55. Fleige S, Walf V, Huch S, Prgomet C, Sehm J, Pfaffl MW. Comparison of relative mRNA quantification models and the impact of RNA integrity in quantitative real-time RT-PCR. Biotechnol Lett. (2006) 28:1601-13. doi: 10.1007/s10529-006-9127-2

56. Seluanov A, Vaidya A, Gorbunova V. Establishing primary adult fibroblast cultures from rodents. J Vis Exp. (2010) 5:2033. doi: 10.3791/2033

Conflict of Interest: $\mathrm{GH}$ owns stock or ownership interests in the following companies: Sangamo Bioscience, Axim Biotechnologies, Juno Therapeutics, Kite Pharma, Novartis, Insys Therapeutics, Abbvie, GW Pharmaceuticals, Cardinal Health, Immunomedics, Endocyte, Clovis Oncology, Cellectis, Aetna, CVS Health, Celgene, Bluebird Bio, Bristol-Myers Squibb/Medarex, crispr therapeutics, IDEXX Laboratories, Johnson and Johnson, Pfizer, Proctor and Gamble, Vertex. GH has operated in a consulting or advisory role for Pfizer, Kite Pharma, Jazz Pharmaceuticals, and Incyte. GH holds research funding from Takeda, Jazz Pharmaceuticals, and Pharmacyclics. GH has accepted travel, accomodations, or expenses from Kite Pharma, Incyte, Pfizer, the Falk Foundation, Jazz Pharmaceuticals, and Astellas Pharma.

The remaining authors declare that the research was conducted in the absence of any commercial or financial relationships that could be construed as a potential conflict of interest.

Copyright (C) 2019 Strattan, Palaniyandi, Kumari, Du, Hakim, Huang, Kesler, Jennings, Sturgill and Hildebrandt. This is an open-access article distributed under the terms of the Creative Commons Attribution License (CC BY). The use, distribution or reproduction in other forums is permitted, provided the original author(s) and the copyright owner(s) are credited and that the original publication in this journal is cited, in accordance with accepted academic practice. No use, distribution or reproduction is permitted which does not comply with these terms. 